\title{
How a Laser Physics Induced Kerr-Newman Black Hole Can Release Gravitational Waves without Igniting the Black Hole Bomb (Explosion of a Mini Black Hole in a Laboratory)
}

\author{
Andrew Walcott Beckwith \\ Physics Department, College of Physics, Chongqing University, Chongqing, China \\ Email: Rwill9955b@gmail.com, abeckwith@uh.edu
}

How to cite this paper: Beckwith, A.W. (2018) How a Laser Physics Induced Kerr-Newman Black Hole Can Release Gravitational Waves without Igniting the Black Hole Bomb (Explosion of a Mini Black Hole in a Laboratory). Journal of High Energy Physics, Gravitation and Cosmology, 4, 743-778.

https://doi.org/10.4236/jhepgc.2018.44042

Received: September 11, 2018

Accepted: October 20, 2018

Published: October 23, 2018

Copyright $\odot 2018$ by author and Scientific Research Publishing Inc. This work is licensed under the Creative Commons Attribution International License (CC BY 4.0).

http://creativecommons.org/licenses/by/4.0/

\begin{abstract}
Note, that micro black holes last within micro seconds, and that we wish to ascertain how to build, in a laboratory, a black hole, which may exist say at least up to $10^{\wedge}-1$ seconds and provide a test bed as to early universe gravitational theories. First of all, it would be to determine, if the mini black hole bomb, would spontaneously occur, unless the Kerr-Newmann black hole were carefully engineered in the laboratory. Specifically, we state that this paper is modeling the creation of an actual Kerr Newman black hole via laser physics, or possibly by other means. We initiate a model of an induced Kerr-Newman black Holes, with specific angular momentum J, and then from there model was to what would happen as to an effective charge, $Q$, creating an $E$ and $B$ field, commensurate with the release of $G W s$. The idea is that using a frame of reference trick, plus $E+i B=-$ function of the derivative of a complex valued scalar field, as given by Appell, in 1887, and reviewed by Whittaker and Watson, 1927 of their " $A$ Course of Modern Analysis" tome that a first principle identification of a $B$ field, commensurate with increase of thermal temperature, $T$, so as to have artificially induced $G W$ production. This is compared in part with the Park 1955 paper of a spinning rod, producing $G W$, with the proviso that both the spinning rod paper, and this artificial Kerr-Newman Black hole will employ the idea of lasers in implementation of their respective $G W$ radiation. The idea is in part partly similar to an idea the author discussed with Dr. Robert Baker, in 2016 with the difference that a $B$ field would be generated and linked to effects linked with induced spin to the Kerr-Newman Black hole. We close with some observations about the "black holes have no hair" theorem, and our problem. Citing some recent
\end{abstract}


suppositions that this "theorem" may not be completely true and how that may relate to our experimental situation. We close with observations from Haijicek, 2008 as which may be pertinent to Quantization of Gravity. Furthermore as an answer to questions raised by a referee, we will have a final statement as to how this problem is for a real black hole being induced, and answering his questions in his review, which will be included in a final appendix to this paper. The main issue which is now to avoid the black hole bomb effect which would entail an explosion of a small black hole in a laboratory. Furthermore as an answer to questions raised by a referee, we will have a final statement as to how this problem is for a real black hole being induced, and answering his questions in his review, which will be included in a final appendix to this paper. In all, the main end result is to try to avoid the so called black hole bomb effect, where a mini black hole would explode in a laboratory setting within say $10^{\wedge}-16$ or so seconds, i.e. the idea would be to have a reasonably stable configuration within put laser energy, but a small mass, and to do it over hopefully $10^{\wedge} 15$ or more times longer than the $10^{\wedge}-16$ seconds where the mini black hole would quickly evaporate. I.e. a duration of say up to $10^{\wedge}-1$ seconds which would provide a base line as to astrophysical modeling of a Kerr-Newman black hole.

\section{Keywords}

Kerr Newman Black Hole, High-Frequency Gravitational Waves (HGW), Causal Discontinuity

\section{Introduction}

Our initial statement of this document, is to use a Kerr-Newman black hole event horizon, with a charge, $\mathrm{Q}$, and a constant angular momentum J, as an induced state of affairs which will be then utilized, if fed by laser induced energy, for the generation of gravitational waves and gravitons. Pursuant to this goal will be utilizing [1] by Ruffini et al. the formation of an event horizon, which will be at the outer boundary of a matter-energy "bubble" of space time, in a laboratory setting, and also utilizing [2] which has a criteria for spatial resolution of a graviton within a confined metric geometry. In addition, we use [3] to generalize the entropy, depending upon graviton production, due to infinite quantum statistics [4] [5], where we assume that graviton count, equivalent to $\mathrm{N}$, i.e. a particle count, is equivalent to an entropy count, for reasons we go into in our manuscript. Furthermore, [6] [7] [8] [9] give background as to the Kerr and Kerr Newman metric used, which is important for our write up, and in addition, we use the non standard treatment of electrodynamics as written up by [10], which is part and parcel of what is implied in [11] and [12]. Note that the treatment of the ergosphere, and the question of a nonzero Angular Momentum, associated with a black hole as given in [13] on page 1283 and 1284 means that we have far more detail as to Black hole physics, than is usually associated with [14], and so 
what we will do, in lieu of [10] is to assume that if we have a complex electric E and magnetic B field associated with a rotating Kerr metric, with charge Q, and with angular momentum $J$ (which we set as a constant in space dimensions due to wanting to keep the complexity of our calculation down), that we employ the trick, as to frame of reference, of setting the complex contributions to the Electrodynamics equation associated with using a frame of reference trick, (plus $\mathrm{E}+$ $\mathrm{iB}=-$ function of the derivative of a complex valued scalar field, as given by Appell, in 1887, [10]). So that if the imaginary part of $E+i B$ vanishes, we then obtain a general magnetic field associated with the rotating Kerr Newman black hole. We do NOT call the angular momentum a constant in time, i.e. we have torque in our model!

In doing so, we use the approximation that to first order that the energy, as given, in this situation, is driven by the usual proportional value of temperature, $\mathrm{T}$, as in standard statistical physics [15], with the temperature, $\mathrm{T}$, driven in part by a laser hitting a target, say of the sort given in Lawrence Livermore implosion pellet experiments. References [16] [17], and [18] pertain to fundamental questions as to the growth of entropy, i.e. why it may start off at absurdly low levels of entropy, as gone over by Penrose, and build up rapidly, why we are considering a laser implosion, and why we are referring to the strength of a signal, of GW, via [13] and its equation 9.51 on its page 505 which has a frequency dependent gravitational wave strain value which we could estimate the role of laser power $\mathrm{W}$, and frequency in our laser experiment.

After this is described, and estimated, we will make reference, to [19] [20], and [21] to describe some of the physics which may be inherent as to a rapid fire laser i.e. [19] is the Park description of how a rotating rod, of a given frequency, $\omega$, of rapid rotation, gives a distinct GW/would be graviton creation if we had the ends of the spinning rod tapped by a laser. This also involves consideration of the type of laser, partly referencing [20] and [21]. The author also had his earlier treatment of this sort of situation in [22] with this present document to be a vastly more refined version of the same idea. [23] [24] [25] [26] add more to the possibility of graviton generation, as to a laboratory created dynamical process, whereas the reference [27] as given by Rindler, on page 154, if we conflate one over the square root of a mass density as greater than the so called horizon of a black hole, a way to tie in the generation of massive gravitons via the spinning rod idea given in [19] (i.e. are GW consistent with gravitons?) with the Kerr black hole.

Having said that, our thought experiment, if it is paired with the inquiry given in references, [19] to [27] as given above, should be tied into resolution of the Equation (26.6) requirement as to the mass $M$, necessary as to the production of a black hole, as given by Peebles [28], which famously has a $1 /(1+z)^{\wedge} 2$ dependence as far as red shift (the greater the red shift, the more likely the creation of black hole. I.e. in effect what we are doing is via laser powered application of energy to an implosion pellet duplicating the idea of formation of primordial 
black holes, and also answering, if we do graviton production right from an induced spinning black hole, what we can expect in relic conditions as far as GW, and gravitons at the start of cosmological expansion.

This should be seen as being in tandem with the idea of the author as given in [29] as to a Tokamak producing high frequency gravitational waves. If we have say $10^{\wedge} 10 \mathrm{~Hz}$, in the Tokamak generation of GW, we are in fact going to the idea of relic high frequency GW, produced at the start of the big bang, and multiply that figure by $10^{\wedge}-26$ to obtain the effect of massive red shifting due to inflation, and a window into the primordial conditions allowing for GW production. At the start of the universe.

In a different way, we can use the idea of an artificial Kerr black hole generating $\mathrm{GW}$, as also another cosmological window into relic conditions, of cosmology.

This, if verified, could be of fundamental importance and will be discussed in our paper. We close with the idea of a causal discontinuity, affecting the production of GW. This last part will be the concluding part of our introduction, and interested readers should access [30], which will be the very last part of our document.

Why reference [30] is important as to our document. In the end, the inquiry about the existence of an artificial Kerr Newman black hole is really about modified gravity. In [31] the three-body problem is analyzed, and in [32] the author submitted a suggestion as to modified gravity, which has been accepted by JHEPGC, and which is really an extension of the ideas given in our document. These in turn are also linkable to what Abraham and Marsden wrote up in pages 663 to 740 in [33].

The idea of modified gravity, so alluded to, should be contrasted with [34] which gives a graviton generation rate for Black holes, on page 45 of [34].

I.e. their rates for emission, as stated by Calmet et al. [34], are for the main part extremely low, except in the case of higher dimensional black holes, as embedded in branes. And, more importantly are for NON rotating black holes, which is different from what we are considering as to the Kerr-Newman induced black hole.

We should keep in mind that there are no specifics given in [34] as to graviton production for rotating black holes, in page 46 , so what we are doing is breaking new ground.

Moreover, if graviton production is, indeed generated by a laser implosion, it allows us to examine relic conditions for early black hole radiation which may allow for analysis of the relationship, if any, between electromagnetics and gravity.

There as of present, no confluence between electromagnetism, and gravity in Einstein's theory of relativity In an alternate modification of GR view this may not be true in the case of origins of nonsingular beginning treatments of initial cosmological conditions as given in [35], and which is elaborated upon in [36]. 
I.e. our inquiry, experimentally may be a way of testing the veracity of these two references, [35] and [36].

Our concluding remarks are in admitting that our inquiry may be a test as to the veracity of the 60 e fold expansion of the universe, as attested through in typical inflation theories, i.e. if inflation is correct, our GW and graviton induced fields are about $10^{\wedge} 26$ times stronger than would be, if inflation had not weakened or dispersed initial gravitational waves, as can be seen by [37].

Finally, our laboratory test, if initiated properly may falsify, or give credence to the 7.7 times $10^{\wedge}-23 \mathrm{eV} / \mathrm{c}^{\wedge} 2$ upper bound to a massive graviton, as reported by Maggiore, in [38], on page 320 which may clarify if there is, say a difference between relic gravitons, and later versions of what gravitons are, well after the onset of inflation.

We assert that a suitable inquiry as to this bound, is in part, an inquiry, once again, into the Mach's principle debate, which is alluded to, in cosmology, in page 167 of Volume 2, of [39], which was in part abandoned by Einstein, in the 1930s. But which is still worth looking at again, pending suitable experimental conditions.

All these topics, and others will be alluded to in part in our inquiry as to the next several pages of our document.

\section{A Brief Recap as to Kerr-Newman Black Hole Physics}

[40] has a complete derivation of how the Lens and Thirring studied the derivation of how a spinning sphere of uniform density created a gravitational field, on page 257 of [40] which leads to a metric of

$$
\begin{aligned}
& \mathrm{d} S^{2}=\left(1-\frac{2 m}{r}\right) \cdot c^{2} \cdot \mathrm{d} t^{2}-\left(1+\frac{2 m}{r}\right) \cdot \mathrm{d} \sigma^{2}+\frac{4 \kappa J}{c^{2} r} \cdot\left(\sin ^{2} \theta\right) \cdot \mathrm{d} \varphi \cdot c \cdot \mathrm{d} t \\
& r \equiv \sqrt{x^{2}+y^{2}+z^{2}} \\
& J=\text { angular momentum of sources } \\
& \mathrm{d} \sigma^{2}=\text { flat space line element } 3 \text { Dim }
\end{aligned}
$$

$$
\begin{aligned}
& \text { Lens Thirring, iff } J=-c^{3} m \frac{a}{\kappa} \\
& \text { and set } r \equiv \rho \\
& \Leftrightarrow \mathrm{d} S^{2}=\left(1-\frac{2 m}{\rho}\right) \cdot c^{2} \cdot \mathrm{d} t^{2}-\left(1+\frac{2 m}{\rho}\right) \cdot \mathrm{d} \sigma^{2}-\frac{4 m a}{c^{2} \rho} \cdot\left(\sin ^{2} \theta\right) \cdot \mathrm{d} \varphi \cdot c \cdot \mathrm{d} t
\end{aligned}
$$

This gives us rotating Kerr

In our consideration, in order to simplify matters, we set $J$ equal to a constant, i.e. this was for ease of calculation and it lead to, with the caveat of, if $a$ is a measure of the angular momentum per mass, and if $m$, in Equation (1) is mass, we can say that $m$ is the "geometric mass" which can lead, to, as given in [40], page 260 with a Coriolis like force given by

$$
\rho \ddot{\varphi}+\left(\frac{-2 m a}{\rho^{3}}\right) \cdot \dot{\rho}=0
$$


If we identify $\rho$ replacing the angular velocity $\omega$, the above is the Coriolis force, as given in page 130 of [40] this will in part, if we add a charge, Q into this business, lead to what is given in [41]

$$
\begin{aligned}
& \mathrm{d} S^{2}=\frac{\tilde{\rho}^{2}}{c^{2}}\left(-\frac{\mathrm{d} r^{2}}{\Delta}+\mathrm{d} \theta^{2}\right)-\frac{\Delta}{\tilde{\rho}^{2}}\left(c \cdot \mathrm{d} t-\tilde{a} \cdot\left(\sin ^{2} \theta\right) \cdot \mathrm{d} \varphi^{2}\right) \\
& \quad-\frac{\left(\sin ^{2} \theta\right)}{\tilde{\rho}^{2}} \cdot\left(c \cdot \mathrm{d} t-\tilde{a} \cdot\left(\sin ^{2} \theta\right) \cdot \mathrm{d} \varphi^{2}\right) \\
& \tilde{a}=J / m \cdot c, \Delta=r^{2}-r_{S} r+\tilde{a}^{2}+r_{Q}^{2} \\
& \tilde{\rho}^{2}=r^{2}+\tilde{a}^{2} \cdot \cos ^{2} \theta \\
& r_{S}=\frac{2 G m}{c^{2}}, r_{Q}^{2}=\frac{Q^{2} G}{4 \pi \varepsilon_{0} c^{4}}
\end{aligned}
$$

We will for the sake of simplicity approximate $J$ as a constant when we do our calculations.

\section{What We Obtain by Using a Charge $Q$ in a Rotating Black Hole Solution}

Reference [42] gives an extremal condition as to the mass of a Kerr Newman black hole, being bounded below, by angular momentum $J$, and charge, $Q$. As given on page 12, of [42] we have that if we have a mass $m$, redefined, as the Christodoulou-Ruffini mass we could set as $M$ by [43] will show the following set of inequalities made equalities. I.e.

$$
\begin{aligned}
& \mathrm{M}=m \\
& S=S_{\text {ext }} \\
& J=J_{\text {ext }} \\
& \text { Then } S=S_{\text {ext }}=\pi \cdot \sqrt{Q_{\text {ext }}^{4}+4 J_{\text {ext }}^{2}} \equiv \pi \cdot \sqrt{Q^{4}+4 J^{2}} \\
& \& \mathrm{M}^{2}=m^{2}=\frac{1}{2} \cdot\left(Q^{2}+\sqrt{Q^{4}+4 J^{2}}\right)
\end{aligned}
$$

The last two parts of Equation (4) can be interpreted using the ideas of Infinite quantum statistics, as a way of making a linkage between entropy, and the counting of numbers of emitted particles, using the relationship given in [4], and [5] of

$$
S=S_{\text {ext }} \approx n(\text { partile count })
$$

We then, can, using Equation (4) and Equation (5) make the following statement as to number of stimulated particles, from a laser hitting an artificial black hole, which we will in this first reading equate with Gravitons (massive) and the matter-energy input into the artificial black hole, i.e.

$$
\begin{aligned}
& \text { If } \mathrm{M}=m, S=S_{\text {ext }} \approx n \equiv(\text { partile count }) \\
& \text { Then } S=S_{\text {ext }} \approx c_{1} n \propto(\text { partile count }) \propto \pi \cdot \sqrt{Q^{4}+4 J^{2}} \\
& \& E_{\text {ext }}=\frac{k_{B}}{2} \cdot T_{\text {applied }} \approx M \cdot c^{2} \\
& \Leftrightarrow\left(\frac{k_{B}}{2 c^{2}} \cdot T_{\text {applied }}\right)^{2}=\frac{1}{2} \cdot\left(Q^{2}+\frac{c_{1} n}{\pi}\right) \\
& \Rightarrow Q^{2}=2 \cdot\left(\frac{k_{B}}{2 c^{2}} \cdot T_{\text {applied }}\right)^{2}+\frac{c_{1} n}{\pi}
\end{aligned}
$$


The particle count, i.e. in this case, stimulated graviton emission from the black hole, and the temperature, $T_{\text {applied }}$ from a laser smashing into a target, will influence an effective charge, $Q$.

\section{Calculation of Electric and Magnetic Fields, for the Kerr-Newman Black Hole, and How One Can Pick a Frame of Reference, Where the E Field Vanishes}

We begin our statement as to looking first at [44], which has a dipole approximation as to a Kerr-Newman black hole, if a charge $Q$ is specified. Then we have a dipole approximation of the following electric field, with $n$, a count of particles per unit area radiated from the artificial Kerr-Newman black hole.

$$
\begin{aligned}
& \vec{E}=\left(\text { Electric field } \simeq \frac{Q}{r^{2}} \cdot \hat{e}_{r}\right) \\
& Q=\sqrt{2 \cdot\left(\frac{k_{B}}{2 c^{2}} \cdot T_{\text {applied }}\right)^{2}+\frac{c_{1} n}{\pi}}
\end{aligned}
$$

What we will do, assuming this base, for the electric field, is to go to [10] where we will have the following electric and magnetic field coupling to consider, namely if we have a potential given by quantity omega in this last equation is similar to the Coulomb potential, except that the radius vector is shifted by an imaginary amount which could lead to the magnetic field given by the following representation.

$$
\begin{aligned}
& \vec{E}+i \vec{B}=-\vec{\nabla} \cdot \tilde{\Omega}=-\vec{\nabla} \cdot \frac{Q}{\sqrt{\left(\vec{r}-i \frac{\vec{J}}{m \cdot c}\right)^{2}}} \\
& \vec{B}=-i \vec{E}+i\left(\vec{\nabla} \cdot \frac{Q}{\sqrt{\left(\vec{r}-i \frac{\vec{J}}{m \cdot c}\right)^{2}}}\right)
\end{aligned}
$$

The approximation which will be used here, is that $J$ is spatially almost invariant (initially) but that it has a distinct function in time, i.e. $J=J(t)$.

I.e. we would be looking at how to have a way to make the following identification which could simply matters, first of all noting that there is, in this situation a B field which is given as in [44] as being approximately real valued with the far distance value of

$$
\vec{B}=\frac{Q\left(\frac{J}{m \cdot c}\right)}{r^{3}} \cdot\left(2 \cdot(\cos \theta) \hat{e}_{r}+(\sin \theta) \hat{e}_{\theta}\right)
$$

If we apply the $2^{\text {nd }}$ part of Equation (8) above, with respect to finding an imaginary part of the $B$ field to be cancelled out, we can write that if we apply Equation (7), Equation (8) and Equation (9) we have if we look at 


$$
\begin{aligned}
& \theta=0 \Rightarrow \vec{B}=\frac{Q\left(\frac{J}{m \cdot c}\right)}{r^{3}} \cdot\left(2 \cdot \hat{e}_{r}\right) \\
& \text { and } \vec{E}=\left(\text { Electric field } \simeq \frac{Q}{r^{2}} \cdot \hat{e}_{r}\right)
\end{aligned}
$$

This is a case where one is having at theta $=0$, both $E$ and $B$ fields, but we can simplify further by muse of

Then,

$$
\begin{aligned}
& E+i B=\frac{\left[-Q \cdot\left(r^{2}-(J / m c)^{2}\right)+Q \cdot(2 i r J / m c)\right]}{\left[\left(\left(r^{2}-(J / m c)^{2}\right)^{2}+(r J / m c)^{2}\right]\right.} \\
& \text { becomes } \\
& i B=\frac{[+Q \cdot(2 i r J / m c)]}{\left[+(r J / m c)^{2}\right]} \Rightarrow B=+2 Q /(r J / m c) ; E=0 \\
& \text { iff } r^{2}=(J / m c)^{2}
\end{aligned}
$$

I.e. we have a vanishing $E$ field in this situation, with a $B$ field with $J=J(t)$, allowing for Torque, which shows up all the time in black hole physics, but we do not have much spatial variation of $J$, the above should be seen as a first order approximation but it is revealing, at the same time, as one is then specifying an axis of rotation in the space-time continuum which contains the artificial, induced Kerr- Newman black hole.

\section{Specifying Conditions for the Production of Gravitons, from the Artificial Kerr-Newman Black Hole}

We can consider working with the induced Kerr-Newman black hole assuming that there is a stimulated emission of particles from the artificial black hole assuming that there is a method of input from lasers, or possibly thermonuclear fusion for input into the formula we will write as

$$
\begin{aligned}
& Q=\sqrt{2 \cdot\left(\frac{k_{B}}{2 c^{2}} \cdot T_{\text {applied }}\right)^{2}+\frac{c_{1} n}{\pi}} \\
& E_{\text {ext }}=\frac{k_{B}}{2} \cdot T_{\text {applied }}
\end{aligned}
$$

We will be examining what would be possible input energy into this "induced Kerr-Newman" black hole.

We go back to optimizing

$$
\begin{aligned}
& Q=\sqrt{2 \cdot\left(\frac{k_{B}}{2 c^{2}} \cdot T_{\text {applied }}\right)^{2}+\frac{c_{1} n}{\pi}} \\
& E_{\text {ext }}=\frac{k_{B}}{2} \cdot T_{\text {applied }} \approx M \cdot c^{2} \\
& 2 M^{2} \geq Q^{2}+\sqrt{Q^{4}+4 J^{2}} \\
& \& 2 M^{2} \geq 2 \cdot\left(\frac{k_{B}}{2 c^{2}} \cdot T_{\text {applied }}\right)^{2}+\frac{c_{1} n}{\pi}+\sqrt{\left[2 \cdot\left(\frac{k_{B}}{2 c^{2}} \cdot T_{\text {applied }}\right)^{2}+\frac{c_{1} n}{\pi}\right]^{2}+4 J^{2}} \\
& \& B=+2 Q /(r J / m c)
\end{aligned}
$$


Note that the expression of $B$, for magnetic field is commensurate with a specific value of $r$, such that we have $E$ effectively disappear.

In this case, we are assuming that, $\mathrm{m}$, in the denominator of $B$, for when $E$ is allegedly zero, is actually M. Going to [45] which restates the problem, to first order we are observing an equality in what is otherwise an inequality,

$$
\begin{aligned}
& 2\left(\frac{B \cdot r \cdot J}{2 c \cdot\left[2 \cdot\left(\frac{k_{B}}{2 c^{2}} \cdot T_{\text {applied }}\right)^{2}+\frac{c_{1} n}{\pi}\right]^{1 / 2}}\right)^{2} \\
& \geq 2 \cdot\left(\frac{k_{B}}{2 c^{2}} \cdot T_{\text {applied }}\right)^{2}+\frac{c_{1} n}{\pi}+\sqrt{\left[2 \cdot\left(\frac{k_{B}}{2 c^{2}} \cdot T_{\text {applied }}\right)^{2}+\frac{c_{1} n}{\pi}\right]^{2}+4 J^{2}}
\end{aligned}
$$

In the case where the above becomes an equality, where there is an extremized value of $r$, we can have that we are observing a situation where a $B$ field, which can be measured, with a value of $J$, for the induced Kerr-Newman metric will lead, to a value of $n$, which in this case would be the number of gravitons emitted by this configured induced Kerr-Metric black hole. And this will be assuming that the temperature $T_{\text {applied }}$ will be created by either a battery of lasers, or by possibly induced fusion.

Our next section will be a description of how to put in $T_{\text {applied }}$ into this system, and we will close with a description of an already worked out protocol for graviton/ GW detection. To do this though we first of all need to understand what allows for GW release from a Kerr-Newman black hole. This is essential, since a count of gravitons so generated and released from this Kerr-Newman black hole, is proportional to the release of information, for reasons we will specify in the next section of our paper. To do this, we consider, both.

\section{What Makes This Paper Possible, a Break Down in the Traditional Blackhole Singularity Block on Information Transfer, and Different Models of How to Put in $\boldsymbol{T}_{\text {applied }}$ into This System, for GW/Gravitons}

First in this treatment is understanding a revolutionary idea, as given in [46] and [47], which can be utilized and explained in the following quote

\section{Quote}

"In particular, if the exterior region of the Kerr family is proven to be dynamically stable-as is widely expected-then it will follow that the $\mathbb{C}^{0}$-Inextendibility formulation of Penrose's celebrated strong cosmic censorship conjecture is in fact false."

\section{End of quote}

The fact that we will discuss graviton release, as information release, will be in; part linked to [48], i.e. a relationship between entropy, and information, which is also based upon $\mathrm{Ng}$, as given in [4] and [5] where entropy is closely linked to 
particle count, but all this depends upon a falsification of [49] [50] [51] [52], which is in fact due that the temperature $T_{\text {applied }}$. may be applied in two specific ways.

First, we may think in terms of a battery of lasers. I.e. see [53] [54] [55] and secondly is due to the idea of applying an underground nuclear explosion as a way to generate sufficient thermal $T_{\text {applied }}$ as given in [56] and [57].

The idea in all of this would be to duplicate in part, say [58], in either laser battery induced implosions, or by kiloton level deep; underground tests sufficient thermal $T_{\text {applied }}$ as to implement use of Equation (14) above. For political reasons, it would be most advisable to go the route of a facility similar in part to the national ignition facility, for obtaining. sufficient thermal $T_{\text {applied }}$ whereas fine tuning the problem of applied magnetic fields taking into account [59].

Crowell, in [60] gave a working summary of what the modification of the singularity mathematics portends to, in a private note which is duplicated below

Quote, from [60]

With the Kerr solution there is behind the interior horizon $r_{-}$-is a timelike region with the singularity. For the Kerr solution the horizon is a ring with closed timelike curves around it. Spacetime is in effect pretty twisted around. However this is a case for the eternal solution, which is a mathematical idealization. This may seem to reflect something unobservable, but in the Penrose diagram below it is the case that a spatial surface in the observable region can by a choice of frame connect with either the other time like region or this odd region. This paper by Defermos and Luk appears to say this region is similar to an exotic four manifold. Exotic or E8 manifolds have no metrizable structure and are not diffeomorphic, though they are homeomorphic. Atiyah, Donaldson. Freedman and Uhlenbeck pioneered this area of mathematics that has some strange implications. The two spatial surfaces are connected by what appears to be a monodromy with the singularity. There is then a connection with ordinary spacetime with this odd spacetime.

End of quote,

This is illustrated in the following diagram which Crowell gave in [60] which is given below which we call Figure 1, which is, in fact fully backed by the following observations which were given to the author by Corda in [61]. Which are in part backed by work Corda did in [62] [63] [64] and [65], whereas much of the ideas are also reflected in the 2017 publication using AdS theory as given in reference [66].

Note that in [61] Corda proceeds with a very logical treatment of a self contained black hole, which has specific limiting cases as to quantum results. We will summarize what Dr. Corda did in the next section of our paper, but before moving forward, it is important to note the confluence of what was done by Kerr, and then earlier by Kurt Godel as given in his rotating model of the Universe as given in [67] [68], which is incidentally often over looked. [67] also makes the point of the inexact nature of what we call singularity theory and black holes. What [67] states is that the Penrose censorship conjecture breaks 


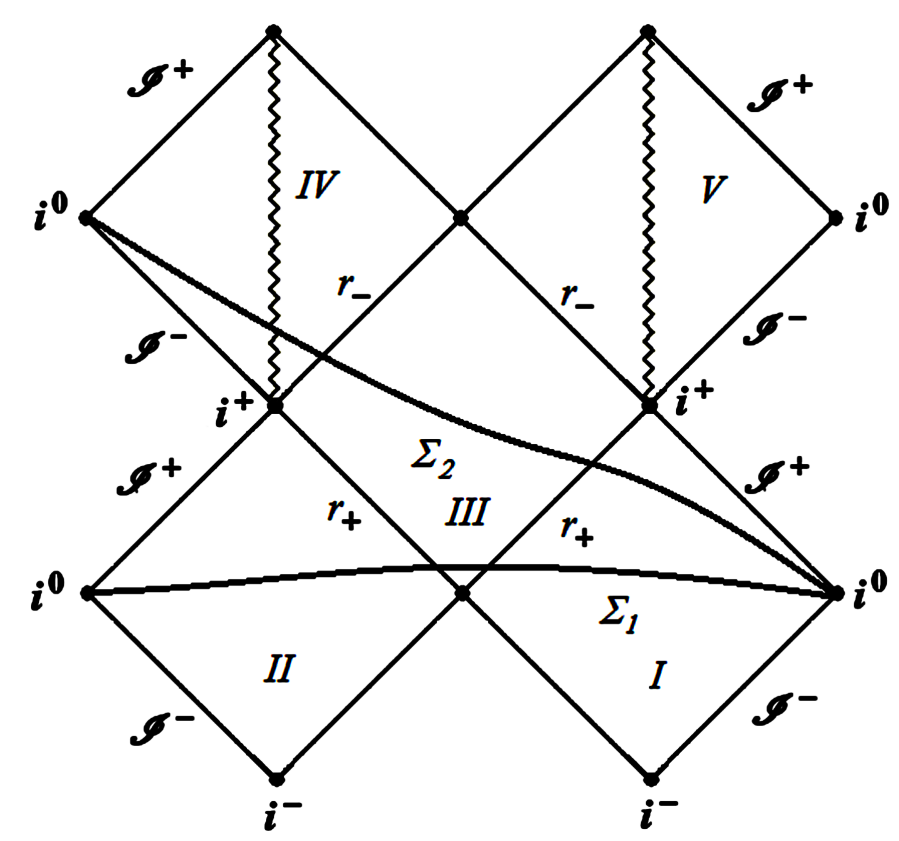

Figure 1. Penrose Diagram supplied by Crowell, in [60] which compliments his information filled observations sent the author in [60].

down, and this is also part and parcel of what we are intending to bring up in our artificial worm hole, of Kerr-Newman type with its charge, $Q$. The rotating universe, as given by Godel,

In [68] we have that, indeed, we can get some links to the Godel spacetime:

See Section 5.7 for a classic discussion of CTCs in the Gödel spacetime. Note, that in Fig. 31, the light cones do indeed tip over, but they also widen, so that vertical coordinate lines are always time-like; indeed, these represent the world lines of the dust particles, so they are time-like geodesics.

In [69] we also have the original Godel paper, which can be looked up as a precursor of the work done by Kerr and Newman, and this is a way also, to intellectually understand the problems inherent in the Penrose censorship conjecture. [70] and also review the issues brought up in [49] [50] [51] and [71]. Having said that, we will address the issues next which Corda raised in [61] about the idea of an effective temperature for black holes, and our comments as to its relationship to our problem.

\section{Comparing Our Work against the Reference [61] Results by Corda as to Effective Temperature for a Black Hole}

In [61] Dr. Corda did an explicit quantum physics analogy as to obtaining an effective temperature $\mathrm{T}$ for black holes which has many similarities as to our results. One aside, one big difference, is that our temperature $T_{\text {applied }}$ involves an applied upon by external temperature regime which we claim would induce conditions for the formation of a Kerr-Newman black hole, whereas what is done in [61] is to assume formation of a black hole leads to the effective temperature, of the black hole itself. I.e. the Corda results as of [61] involve an indi- 
genous temperature for the black hole, which is created in the process of formation of the black hole. I.e. the [61] result does not explicitly assume creation of the black hole in question due to application of an external temperature, of the sort we did in our $T_{\text {applied }}$. Having said that, many of the results of [61] are, in part, extremely close to ours, and we can use [61] as a way to ascertain the degree of proximity to quantum processes, which is the main benefit of the analysis given in [61] by Corda.

In a word, [61] delineates a careful analysis of how much quantum process contribute to Black Hole entropy, which we also look at, and in turn is related to the temperature, $\mathrm{T}$, which Corda derives in [61].

In [61] Corda delineated the effective temperature of the black hole in question as

$$
\begin{aligned}
& T_{E}(\omega) \equiv \frac{1}{4 \pi(2 \tilde{M}-\omega)} \\
& \& \tilde{N}=\# \text { of quanta } \\
& \tilde{M} \approx \text { Black hole mass } \\
& \omega=\text { frequency emitted radiation } \\
& \& S_{\text {Total }} \underset{\text { full quantization }}{\longrightarrow} 2 \pi \tilde{N}+\left(\frac{3}{2 \pi \tilde{N}}-\ln 2 \pi \tilde{N}\right)
\end{aligned}
$$

If so, and we assume full quantization is achieved in our model of the quantum black hole idealization we can make the following identification. I.e. to make 15 consistent with our results we can do the following, i.e. assume that $n=$ number of gravitons is approximately the same as the number of quanta, I.e. state that we can have an overlap between the results of [61] with our results if the following block of equations is utilized? I.e. in a word, the quantity $T_{E}(\omega) \equiv \frac{1}{4 \pi(2 \tilde{M}-\omega)}$ as an effective temperature for the black hole being formed, is added as a would be definition, separate from the applied external temperature $T_{\text {applied }}$ which presumably would be put into the formed Kerr-Newman black hole. I.e. we make the following block of equations to be considered as the main result of this section of our paper.

$$
\begin{aligned}
& T_{E}(\omega) \equiv \frac{1}{4 \pi(2 \tilde{M}-\omega)} \& \tilde{N}=\# \text { of quanta } \\
& \tilde{M} \approx \text { Black hole mass } \\
& \omega=\text { frequency emitted radiation } \\
& \& S_{\text {Total }} \underset{\text { full quantization }}{\longrightarrow} 2 \pi \tilde{N}+\left(\frac{3}{2 \pi \tilde{N}}-\ln 2 \pi \tilde{N}\right) \text { Corda result } \\
& \quad S \underset{\text { full quantization }}{\longrightarrow} c_{1} \cdot n \text { My result }
\end{aligned}
$$

Question, can we pick $c_{1}$ such that

$$
c_{1} n \equiv 2 \pi \tilde{N}+\left(\frac{3}{2 \pi \tilde{N}}-\ln 2 \pi \tilde{N}\right) \text {, if } n=\tilde{N} ?
$$


If we can satisfy Equation (16) above, we then come to a very fundamental question for our inquiry which is as follows and will be briefly mentioned as framing one of the big questions this manuscript will raise, namely.

\section{What Are Conditions Permitting}

$$
T_{E}(\omega) \equiv \frac{1}{4 \pi(2 \tilde{M}-\omega)} \cong T_{\text {applied }} ?
$$

We submit that this is not a trivial question and answering it would lead to perhaps successful implementation of our idea as to forming a Kerr-Newman artificial black hole. To answer it will require well posed modeling and experimental constraint conditions which we will try to bring up in this section VIII.

First of all, to do this identification of $T_{E}(\omega) \equiv \frac{1}{4 \pi(2 \tilde{M}-\omega)} \cong T_{\text {applied }}$, we have to have the fix put in as far as Equation (16). This is basic. Secondly, is to investigate the forwarded to inquiry as given to the author by Lawrence Crowell, August 30, 2019, namely [72].

We can create a sort of artificial black hole by recognizing that the Weyl tensor $C_{-}\{a b c d\}$ defines symmetric 2 -tensor components $E_{-}\{a c\}=g \wedge\{b d\} C_{-}\{a b c d\}$ that are analogous to the electric field. That this is a rank 2 tensor means there are two polarization directions. The Hodge star or with Levi-Civita you can form the magnetic field analogue. With Bern and Dixon we have the phenomenological analogue between gravitation and gauge fields where a rank 2 tensor of this form may be formed by the entanglement of two gauge boson in a triplet state. So gluons can define a "sort of graviton" and for $S U(4)-->S U(2,2)$ under an $S T U$ duality transformation this extended $Q C D$ has some duality with gravitation. $S U(2,2) \sim S O(4,2)$ is the isometry group for AdS_5. It is not hard to work out the roots and weights of the $S U(4)$, where it has an additional weight vector and 6 additional charges. Standard SU(3) QCD embeds into this theory. How SU(4) works in standard model or GUT physics is hard to know, but I think the 6 additional vector terms may form entanglements in singlet states that are the 3 Goldstone bosons of the Higgs field and the remaining weight with its anti-color field may form the left over Higgs particle $h$ that was detected in 2012.

So in this way the heavy ion physics of the LHC with the A Large Ion Collider Experiment (ALICE) there is with the lead ions an atomic weight times the $13 T e V$ of energy, which forms a quark-gluon plasma at considerable energy. The ALICE work is a bit of the forgotten last child in the LHC experiments, but in many ways it is just as interesting as proton collisions. This should form something analogous to a black hole. The decay of this results in gluon pairs that should have analogues with gravitational waves.

I.e. a mathematical investigation may, indeed yield conditions in which one can establish $T_{E}(\omega) \equiv \frac{1}{4 \pi(2 \tilde{M}-\omega)} \cong T_{\text {applied }}$. Furthermore, is to also investigate 
if we can have an investigation of the strength of gravitational waves, as discussed in [13], as given on page 505 of [13] formula for GW "strain", namely in the case of laser light implosion, as on page 505 of [13], we have $h \sim$ strain strength of GW which may be measured from an induced black hole, as given by formulas from [13] which were initially for a laser interferometer system, in LIGO, with the following comparisons, i.e. looking at

$$
\begin{aligned}
& T_{E}(\omega) \equiv \frac{1}{4 \pi(2 \tilde{M}-\omega)} \cong T_{\text {applied }} \\
& \text { if } \omega=\omega_{G W} \\
& \tilde{M}=\text { 'mass induced black hole } \\
& \omega_{\text {Laser light }}=\text { frequency of laser } \\
& W_{\text {Laser light }}=\text { Laser light power } \\
& h \sim \mathrm{GW} \text { strength }=\left(\frac{\hbar \omega_{G W}}{4 \pi \omega_{\text {Laser light }} W_{\text {Laser light }}}\right)^{1 / 2}
\end{aligned}
$$

\section{What Equation (17) Portends for Emitted GW (Graviton?) Radiation from the Artificial Black Hole}

This Equation (17) is for laser induced implosions on a black hole, Kerr-Newman style, which would in the case of the national ignition facility have an enormous power behind this, and this assumes a signal to noise ratio of about 1 . Note this Equation (17) in [13] was originally for laser interferometry in a LIGO style system, and to get what we are seeking, we are likely assuming that the laser light would be very high frequency and that we would be that both $\omega_{\text {Laser light }}$ and $\omega_{G W}$ for frequency of emitted GW would be very high, likely in the $10^{\wedge} 8$ to $10^{\wedge} 12 \mathrm{~Hz}$ range.

Furthermore as far as the size of the induced Kerr Black hole we would be looking at an induced ring singularity of at least an angstrom in "width" i.e. likely much larger.

Our working assumption would be then that the emitted GW from the "induced black hole "would scale roughly as, if

$$
\begin{aligned}
& \omega_{G W} \approx 2 \tilde{M}-\frac{1}{4 \pi\left(T_{\text {applied }}\right)} \\
& \text { if } \omega=\omega_{G W} \\
& \tilde{M}=\text { 'mass induced black hole }
\end{aligned}
$$

This should be seen against the usual dimensional analysis, assuming that $k_{B}=\hbar=c \rightarrow 1$ in dimensional analysis which would be seen as akin to the more usual [15]

$$
E_{\text {ext }}=\frac{k_{B}}{2} \cdot T_{\text {applied }} \approx \tilde{M} \cdot c^{2} \underset{k_{B}=\hbar=c \rightarrow 1}{\longrightarrow} \omega_{G W}
$$

I.e. the higher one is getting to a huge applied temperature we would be looking at a system approaching Equation (19). 
In addition, if we are referring to a ring singularity [1] in an induced Kerr-Newman black hole, we would have say [73]

$$
\begin{aligned}
& \lambda_{G W} v_{G W} \equiv 2 \pi \lambda_{G W} \omega_{G W} \approx c \equiv 1 \\
& \Rightarrow \lambda_{G W} \approx 1 / 2 \pi \omega_{G W} \\
& \text { If } \lambda_{G W} \geq \text { Radius of Ring } \\
& \text { Radius of Ring } \approx 1 / 2 \pi \omega_{G W}
\end{aligned}
$$

If the radius of the (black hole Singularity) ring, is not on an angstrom scale, it is easy to postulate that one is having at least a $10^{\wedge} 10 \mathrm{~Hz}$ frequency, in emitted radiation, and the strength of the GW, can be easily made, with adjustment in input parameters, so $h \sim 10^{\wedge} 23$ is probable. I.e. this should be seen in the light of having a suitable applied temperature $T_{\text {applied }}$ applied to the artificial Kerr-Newman black hole provided that we are looking at, say

$$
h \sim \mathrm{GW} \text { strength }=\left(\frac{\hbar \omega_{G W}}{4 \pi \omega_{\text {Laser light }} W_{\text {Laser light }}}\right)^{1 / 2} \propto 10^{-23}
$$

Keep in mind, that we are considering how to come up with GW and graviton signals which could be experimentally tested, which is why we are writing up our next section.

\section{Comparison of Our Idealized Experiment with a Rotating Rod for Generation of GW and Gravitons}

In doing this, we are assuming here that we can look at [19] [20], and [21], which is in effect considering [74] and [75].

We argue that in effect we have something similar to a rotating rod, as far as the physics of GW, but without the problems inherent in merely applying a laser system to the end of a rod.

The bridge between the rotating rod, and the Kerr-Newman black hole would lie in the idea of $J=J(t)$. I.e. that we could induce torque, in this problem, i.e. like a spinning top, but to definitely allow us to examine methods of GW release, say as of the early universe, which was brought up in [76], i.e. if we have small Kerr Newman black holes, at an early date, and this interlocks with quantum effects, we may be in a position as to understand [74] and [75] issues as far as quantum qubits and other information theory links of how black holes may contain quantum information which plays a role in cosmological evolution.

More to the point as of [19] [22], and [23] we would be avoiding some very practical problems which are in the idea of a spinning rod, which is what sort of material could possibly withstand the onset of extreme laser heat hitting the ends of a rotating rod, and also the issue of generation of stochastic noise, I.e. the old signal to noise ratio problem, in terms of what we could expect if a laser timed as having pulses down to $10^{\wedge}-9$ second intervals firing and hitting the ends of a spinning laser rod.

In principle, this could be overcome, but in practicality, it would involve problems like scattering of laser light hitting the end of a rotating rod. 
Again, in principle, with sufficiently refined engineering, assuming a fantastically well synchronized laser, with say up to laser shots of down to $10^{\wedge}-9$ seconds, one could get a GW wave signal and satisfy all the issues inherent in [19] [22], and [23], without generating tons of stochastic noise.

This author doubts it.

The second requirement would be in having duration of a process of GW generation say of up to at least 10 or so seconds.

Meaning for $10^{\wedge}-9$ separation of time from one laser shot hitting the end of a spinning laser rod, we would have 10 BILLION laser shots.

As a practical matter, the author does not see how this could possibly be done. The author would be happy to be wrong, but this is precisely why the author went to the idea of an induced rotating Kerr-Newman black hole, where the existence and dynamics of a $B$ (magnetic) field would hopefully induce torque into the system, so as to avoid this experimental issue brought up.

Now that we have done this, we wish to discuss issues as connected to GW generation and our would be artificial Kerr-Newman black hole model.

In doing so, we also invoke use of reference [77] which is the black hole bomb and which is central to our analysis.

\section{Considering Now the Issue of How to Possibly Detect High Frequency GW in This Problem. And Why We Would Like to Avoid the Problem of Super Radiance for Our Would Be an Artificial Kerr Newman Black Hole}

From [77] we have the following quote:

$A$ wave impinging on a Kerr black hole can be amplified as it scatters off the hole if certain conditions are satisfied giving rise to superradiant scattering. By placing a mirror around the black hole one can make the system unstable. This is the black hole bomb of Press and Teukolsky. We investigate in detail this process and compute the growing timescales and oscillation frequencies as a function of the mirror's location. It is found that in order for the system black hole plus mirror to become unstable there is a minimum distance at which the mirror must be located. We also give an explicit example showing that such a bomb can be built. In addition, our arguments enable us to justify why large Kerr-AdS black holes are stable and small Kerr-AdS black holes should be unstable.

Hence, we wish to avoid the "super radiance bomb".

If we do so, then we have the situation as described that the small Kerr Newman black hole will NOT be unstable, but which can be actually measured. How can we do this?

Fortunately, Dr. Li, Fangyu, and Dr. Hao Wen of Chongqing University have equipment which may be up to the problem of $10^{\wedge} 10 \mathrm{~Hz}$ or higher laboratory measurements of GW, and we wish to refer interested readers into looking at [78], and this is to find a way to measure in a laboratory the ejected gravitons which our artificial Kerr Newman black hole would be generating.

As to the interior to exterior version of the Kerr black hole, what we are doing 
is in essence, the traversing of quantum information across a causal barrier of space time. But in a manner which contravenes the problem given in [77]. I.e. we are showing how to avoid instability in our manufactured Kerr Newman black hole so we can come up with experimental conditions allowing for the detection of GW.

In doing so, we are, in effect crossing a causal structure boundary, how why do we bring this up?

In [79] Dowker outlines the essential issue, i.e. the Kerr Newman black hole is in essence the boundary of what can be called traditional Causal structure. To a degree, this involves what was set up in [80] i.e. we are creating by the superposition of external conditions the prototype of the something from nothing program, as referenced in [80] but we do it with regards to external applications of energy into our Kerr Newman structure. The issue is a cross between the mathematics described in [81], which is, if we conflate the similarities between entropy structure in the start of our universe, with black holes as given by Lousto et al., in [3], is a way of saying our external application of energy, leading to graviton production from the Kerr Newman black hole we wish to create, is similar to the flow of information problem we are outlining in the evolution of cosmological structure, through this problem.

As to information, in XII, we outline an extension of Seth Lloyds information and computational evolution of the universe. I.e. we are through our black hole experiment, leading up to a possible test of the hypothesis given in XII

Even if we do not kill off superradiance, in black hole production, though our experiment in the laboratory, we may be able to get a bound on the admitted upper bound to massive gravitons.

In doing this, we are coming up with a model as to small black holes producing gravitons and information. However, if we cannot falsify Super radiance, we need to look at [82] giving at least an upper bound to the mass of a graviton. And to consider the situations given in [83] [84] [85] [86] and [87].

This also may allow us to come up with a massive graviton version of the Calmert document, as to quantum black holes, as cited in [34]. The cited result as of page 45 of [34] is for massless gravitons, and in house laboratory experiments may allow us to expand this to the massive graviton case.

Having said this, we will go to the Quantum information case, next which we regard as a way to quantify an output from the Kerr Newmann black hole we have discussed so far.

\section{Seth Lloyd's Universe as a Quantum Computer Model with Modifications}

We use the formula given by Seth Lloyd (2002) [48] that defines the number of operations the "Universe" can "compute" during its evolution. Lloyd (2002) [48] uses the idea attributed to Landauer that the universe is a physical system with information processed over its evolutionary history. Lloyd also cites a prior paper where he attributes an upper bound to the permitted speed a physical system can have in performing operations in lieu of the Margolis/Levitin theorem. He 
specifies a quantum mechanically given upper limit value (assuming $\mathrm{E}$ is the average energy of the system above a ground state value), obtaining a first limit of a quantum mechanical average energy bound value of

$$
\text { [\# operations/sec }] \leq 2 E / \pi \hbar
$$

The second limit to this number of operations is strictly linked to entropy, due to considerations of limits to memory space, which Lloyd writes as

$$
\text { [\# operations] } \leq S(\text { entropy }) /\left(k_{B} \cdot \ln 2\right)
$$

The third limit, based on strict considerations of a matter-dominated universe, relates the number of allowed computations (operations) within a volume for the alleged space of a universe (horizon). Lloyd identifies this space-time volume as $c^{3} \cdot t^{3}$, with $c$ the speed of light, and $t$ an alleged time (age) for the universe. We further identify $E$ (energy) $\sim \rho \cdot c^{2}$, with $\rho$ as the density of matter, and $\rho \cdot c^{2}$ as the energy density (unit volume). This leads to

$$
[\text { \# operations } / \mathrm{sec}] \leq \rho \cdot c^{2} \times c^{3} \cdot t^{3}
$$

We then can write this, if $\rho \sim 10^{-27} \mathrm{kil} / \mathrm{meter}^{3}$ and time as approximately $t \sim 10^{10}$ years. This leads to a present upper bound of

$$
\text { [\# operations] } \approx \rho \cdot c^{5} \cdot t^{4} \leq 10^{120}
$$

Lloyd further refines this to read [48]

$$
\text { \# operations }=\frac{4 E}{\hbar} \cdot\left(t_{1}-\sqrt{t_{1} t_{0}}\right) \approx\left(t_{\text {Final }} / t_{P}\right) \leq 10^{120}
$$

We assume that $t_{1}=$ final time of physical evolution, whereas $t_{0}=t_{P} \sim 10^{-43}$ seconds and that we can set an energy input by assuming, in early universe conditions, that $N^{+} \neq \varepsilon^{+} \ll 1$ and $0<N^{+}<1$. So that we are looking at a graviton-burst-supplied energy value of

$$
E=\left(V_{4 \text {-Dim }}\right) \cdot\left[\rho_{V a c}=\frac{\Lambda}{8 \pi G}\right] \sim N^{+} \cdot\left[\rho_{\text {graviton }} \cdot V_{4 \text {-vol }} \approx \hbar \cdot \omega_{\text {graviton }}\right]
$$

Furthermore, assuming the initial temperature is within the range of $T \approx 10^{32}-10^{29}$ Kelvin, we have a Hubble parameter defined along the route specified by Lloyd [48]. This is in lieu of time $t=1 / H$, a horizon distance defined as $\approx c / H$, and a total energy value within the horizon as

$$
\text { Energy (within the horizon) } \approx \rho_{C} \cdot c^{3} /\left(H^{4} \cdot \hbar\right) \approx 1 /\left(t_{P}^{2} \cdot H\right)
$$

And this for a horizon parameter Lloyd (2002) defines as [48]

$$
H=\sqrt{8 \pi G \cdot\left[\rho_{\text {crit }}\right] / 3 \cdot c^{2}}
$$

And a early universe

$$
\rho_{\text {crit }} \sim \rho_{\text {graviton }} \sim \hbar \cdot \omega_{\text {graviton }} / V_{4-\mathrm{Vol}}
$$

Then

$$
\begin{aligned}
\text { \# operations } & \approx 1 /\left[t_{P}^{2} \cdot H\right] \approx \sqrt{V_{4-\mathrm{Vol}}} \cdot t_{P}^{-2} / \sqrt{\left[8 \pi G \hbar \omega_{\text {graviton }} / 3 c^{2}\right]} \\
& \approx[3 \ln 2 / 4]^{4 / 3} \cdot\left[S_{\text {Entrophy }} / k_{B} \ln 2\right]^{4 / 3}
\end{aligned}
$$

We state specifically that this should be considered as far as information, which is also linked to Entropy which is the actual cornerstone of this model as 
far as output as to the Kerr Newmann black hole.

Having said, that we ascertain that the next stop for a review of what we are doing is to also give a recap of the black hole has no hair idea This black hole has no hair is intimately related to the ideas given in [88]-[98], as background, and we then present this concept for our placing of what we will say finally as far as gravity.

\section{The Black Hole Has No Hair Conjecture and the Future of Information Theory Connected with Black Hole Physics}

In examining this supposition, we first ask the readers to consider the supposition that black hole solutions of the Einstein-Maxwell equations of gravitation and electromagnetism can be characterized by only three externally observable classical parameters: mass, electric charge, and angular momentum. And that all other information of space time "matter-energy" will then thereby disappear behind the event hole horizon and is not accessible to external observers. This is brought up in [99] even in the case of positive cosmological constants, and is applicable as to the situation given in [100]. Even if we have a modified situation of brane world physics, we can then use [101] and [102], which is also linked to [103] by Carroll et al.

Note that in [104] we appear to have two ways out of this conjecture. Non Abelian structure, or curvature in higher dimensional space time.

This is what will be sought out to be confirmed, or falsified in our investigations. Note that Israel established the following, from [105], page 20 of this link.

Quote:

The Israel Theorem This celebrated theorem establishes that all static black hole solutions of Einstein's vacuum equations are spherically symmetric [20]. Israel was able to obtain this result \{and its extension to electrovac spacetimes \{by considering a particular foliation of the static 3-dimensional hypersurface $\Sigma$ \}.

This reference [20] in [105] is actually our [106] which has the following abstract.

The following theorem is established. Among all static, asymptotically flat vacuum space-times with closed simply connected equipotential surfaces g00 = constant, the Schwarzschild solution is the only one which has a nonsingular infinite-red-shift surface g00 $=0$. Thus there exists no static asymmetric perturbation of the Schwarzschild manifold due to internal sources (e.g., a quadrupole moment) which will preserve a regular event horizon. Possible implications of this result for asymmetric gravitational collapse are briefly discussed.

We do not have, in our Kerr Newman black hole anything remotely spherically symmetric, and in fact, the impingement of external laser beams, or say an explosion generated by atomic weapons, or other means would definitely lead to anything but spherical symmetry, nor could we expect to have the highly simplified version of black hole mass, as given in [105] via what is called on page 23 of [105]. An Electrovac Bogomol'nyi Equation which is given as Equation (12.1) of page 23 yields 


$$
\begin{aligned}
& \mathrm{M}^{2}=\left[\frac{\kappa \mathrm{A}}{4 \pi}\right]^{2}+\mathbb{Q}^{2}+\mathrm{P}^{2} \\
& \mathrm{M}=\text { black hole mass } \\
& \mathrm{A}=\text { surface area } \mathrm{BH} \\
& \mathbb{Q}=\text { electric charge } \\
& \mathrm{P}=\text { Magnetic charge } \\
& \Rightarrow T_{H}=\text { Hawking temp }=\frac{2}{\mathrm{~A}} \cdot \sqrt{\mathrm{M}^{2}-\mathbb{Q}^{2}-\mathrm{P}^{2}}
\end{aligned}
$$

In the situation we outlined for our problem, we do not have spherical symmetry, and our electric field is missing so as to approximately have initially no real electric field contribution so to first order we would write instead a modification of Equation (12.1) of page 23 of [105] where we are still assuming 21. Here we present a systematic approach to divergence identities for electrovac black hole configurations with nonrotating horizon

$$
\begin{aligned}
& \mathrm{M}^{2}=\left[\frac{\kappa \mathrm{A}}{4 \pi}\right]^{2}+\mathbb{Q}^{2}+\mathrm{P}^{2} \\
& \mathrm{M}=\text { black hole mass } \\
& \mathrm{A}=\text { surface area } \mathrm{BH} \\
& \mathbb{Q}=\text { electric charge }=0 \\
& \mathrm{P}=\text { Magnetic charge } \\
& \Rightarrow T_{H}=\text { Hawking temp }=\frac{2}{\mathrm{~A}} \cdot \sqrt{\mathrm{M}^{2}-\mathrm{P}^{2}}
\end{aligned}
$$

In a net sense, this would raise the effective Hawkings temperature and then we would compare this with the Equation (17) values given earlier.

Equation (33) is assuming a very high level of symmetry, or near symmetry, whereas Equation (3) has a temperature dependence given by conditions not necessarily dependent upon symmetry whereas we do not have to have a non rotating horizon, and we then have to compare

$$
\begin{aligned}
& \mathrm{M}^{2}=\left[\frac{\kappa \mathrm{A}}{4 \pi}\right]^{2}+\mathbb{Q}^{2}+\mathrm{P}^{2} \\
& \mathrm{M}=\text { black hole mass } \\
& \mathrm{A}=\text { surface area } \mathrm{BH} \\
& \mathbb{Q}=\text { electric charge }=0 \\
& \mathrm{P}=\text { Magnetic charge } \\
& \left.\Rightarrow T_{H}=\text { Hawking temp }=\frac{2}{\mathrm{~A}} \cdot \sqrt{\mathrm{M}^{2}-\mathrm{P}^{2}} \text { (for non-rotating } \mathrm{BH}\right) \\
& \quad \text { versus } \\
& T_{E}(\omega) \equiv \frac{1}{4 \pi(2 \tilde{M}-\omega)}(\text { for rotating } \mathrm{BH}) \\
& \omega=\text { emitted radiation frequency } \\
& \tilde{N}=\# \text { of quanta } \\
& \tilde{M} \approx \text { Black hole mass } \\
& \omega=\text { frequency emitted radiation }
\end{aligned}
$$

The mass dependence in these situations, corresponding to rotating and non 
rotating black holes, and the respective temperatures are completely different.

I.e. we argue that in the case of not necessarily close to spherically symmetric, rotating black holes, as we have outlined, that we may via the arguments given in [105] be seeing marked differences. The differences between the top and bottom temperature dependences which show up here have to be investigated as to their fundamental import and meaning, in our research work.

This will be incorporating ideas which will fundamentally need [106], and [107] and [108] and [109] to implement if we are looking at Ergodic Mixing, the idea of initial black holes, and perhaps a multiverse, as a leavening influence of entropy and early universe conditions, as well as black hole physics.

\section{Conclusion: A Lot to Do and How Decoding the Essence of GW Radiation in the Laboratory May Help Us Get It Done}

The main issue is how to create an actual black hole in a laboratory as to how to do it, without igniting the so called "black hole bomb" as related to in.

In [88]-[93] we currently have a lot of model related experimental work to consider, but we do not really have a consistent theory of gravity yet. This is also to move past the Author's presentation as given in [81]. I.e. in effect, our level of knowledge is equivalent to when Feynman outlined the Parton model [94] [95]. We have excellent phenomenalistic models, but fail to get to the essence of why we see what we do, in many gravitational physics situations.

Aside from necessary engineering work to do, if any of what we are trying to do is achieved, we can follow up on a suggestion made by Dr. Crowell as to this paper, "The interior of dynamical vacuum black holes" in [47].

As time permits, this author recommends following up on the suggestion made by Dr. Crowell in organizing a study group to go through this entire 217 page masterpiece.

Something along these lines will be organized, and in doing so, the essence of information transfer in and out of black holes should be analyzed as well as the essence of decoding what is meant by the cosmological singularities purportedly associated with Black holes.

Note our Appendix B also brings up the possibility, as first alluded to in [96] about the breakdown of the so called "black holes have no hair" conjecture.

Note that [96] has, in its introduction.

Quote

We first show that the standard black hole no-hair theorem underlying this belief, although true in the abelian setting, does not necessarily extend to the non-abelian case. This indicates the possibility of solutions with non-trivial gauge and Higgs configurations decaying exponentially $\{\mid$ it outside\} the horizon. We then find such solutions by numerical integration of the classical equations for the case of $S U(2)$ coupled to a Higgs doublet (the standard model less hypercharge).

I.e. if there was a break in this no hair theorem (conjecture) it likely has to do 
with an equivalent development allowing for non abelian situations in our formulation of a problem concerning Kerr-Newman black holes.

Crowell, in [97] specifically alluded to having $E=0$ and $B$ not equal to zero, with respect to black hole physics, as breaking of the "No hair conjecture" of black holes. The author does not deny it, and also that as Crowell correctly noted, that the E and B fields as used in this manuscript are for electromagnetic fields far from the Kerr Newman black hole.

I.e. what was done is the leading order of electromagnetic field contributions, and the use of the magnetic field as the preferred venue is for, frankly, helping to allow for magnetic field to help induce torque, in the Kerr-Newman black hole, so we can have rotation, which is making our problem then akin to using reference [5] as cited by [96] plus the caveat offered in [96] which is called [6] which we quote, as actually being reference [98].

From [98].

Quote

We describe in detail two different types of black hole hair that decay exponentially at long range. The first type is associated with discrete gauge charge and the screening is due to the Higgs mechanism. The second type is associated with color magnetic charge, and the screening is due to color confinement.

Appendix B summarizes what can be said about the typical Black hole have no hair, idea, and also a simple suggestion as to how and why our problem may contravene this Conjecture (theorem?).

We note that in all of this we are in effect reviewing what was brought up in page 95 of [98].

Quote

Another, weaker but more profound way of interpreting the no-hair theorems is as statements about the classification of stationary black holes. According to this weaker interpretation, the properties of a black hole are completely determined, within any given theory, by the value of its mass, angular momentum, and continuous gauge charges. As we have seen, this weaker interpretation is violated non-perturbatively in $\hbar$, by discrete gauge hair. This form of hair expands the space of states of black holes. It is therefore appropriately called primary hair.

Reference [98], like [47] is a huge reference. Aside from reviewing [47] in a study group, this author will also recommend that in the non quantum case of black hole physics that some serious thought be given to the idea of non abelian structures which may encompass our experimentally induced Kerr-Newman black hole, whereas also, then, would be a review of if there is some evidence, emerging, in a quantum black hole case for the existence of "discrete gauge hair" which may indeed permit non abelian structure, if we are lucky or at least be consistent with the write up given by Coleman et al. for [98].

If both these approaches fail, we still can gain major benefits from a concerted study as to each of the chapters of [47].

Keep in mind, in all of this, that we are NOT abandoning in a conventional 
sense the possibility of a non zero E field in our experimentally induced KerrNewman black hole. As noted by Crowell, the E and B fields as referenced are for far field approximations.

We may wish to do, in a future date, in a study of Q, what we would expect from more detailed E and B fields connected to the Kerr-Newman black hole.

This will of course present difficulties, but keep in mind that if the experimental apparatus for measuring gravity and gravitons is meters away from the induced Kerr-Newman black hole whereas the "event horizon" of the Kerr Newman black hole, would be MUCH smaller, that up to a good approximation we are indeed in a far field zone experimentally.

This will present many difficulties.

Finally we ascertain an entry from [107] which has the following area of a black hole (rotating) similar to the Kerr-Newman black hole surface area which is in turn compared to an earlier version of 2 times the square of mass, so obtained. With a value of $\mathrm{Q}$, as a "topological charge" earlier thrown in.

$$
\begin{aligned}
& 2 M^{2}=\frac{1}{2} \cdot\left(\frac{4 \pi}{A}\right) \cdot\left[\left(\frac{A}{4 \pi}+Q^{2}\right)^{2}+4 J^{2}\right] \\
& 2 M^{2} \approx 2 \cdot\left(\frac{k_{B}}{2 c^{2}} \cdot T_{\text {applied }}\right)^{2}+\frac{c_{1} n}{\pi}+\sqrt{\left[2 \cdot\left(\frac{k_{B}}{2 c^{2}} \cdot T_{\text {applied }}\right)^{2}+\frac{c_{1} n}{\pi}\right]^{2}+4 J^{2}} \\
& \& Q=\sqrt{2 \cdot\left(\frac{k_{B}}{2 c^{2}} \cdot T_{\text {applied }}\right)^{2}+\frac{c_{1} n}{\pi}}
\end{aligned}
$$

From here, we can then if we equate the $2^{\text {nd }}$ and third lines of Equation (35) we could, numerically ascertain a value of the term, $T_{\text {applied }}$, while comparing this with the first line of Equation (35) above. In doing so we would call this value of $T_{\text {applied }}$ as $\left(T_{\text {applied }}\right)_{\text {derived }}$ so that

$$
\begin{aligned}
& T_{\text {applied }} \underset{\text { derived }}{\longrightarrow}\left(T_{\text {applied }}\right)_{\text {derived }} \\
& \Leftrightarrow(\Delta E)_{\text {derived }}=\frac{k_{B}}{2} \cdot\left(T_{\text {applied }}\right)_{\text {derived }} \approx \frac{\hbar}{\left(\Delta t_{\text {applied }}\right)_{\text {derived }}} \approx \hbar\left(\omega_{\text {applied }}\right)_{\text {derived }}
\end{aligned}
$$

If this is done and we then wind up with $\left(\omega_{\text {applied }}\right)_{\text {derived }}$ on the order of $10^{\wedge} 10$ $\mathrm{Hz}$, with an inverse relationship roughly of the size of $10^{\wedge} 10$ seconds, with the $\mathrm{n}$, above, as given by gravitons being counted, and with $c_{1} n$ set by Equation (16) and Equation (17) we are then in terms of experimental input well on our way toward setting parameterization of a quantum theory of gravity which so far has eluded experimentalists and theorists.

This in its own way would be a follow up of what has been presented and marrying our work with the insights as given by Christian Corda which we have ascertained and used. It also sets the stage for utilization of [108] in a follow up which will, among other things, address the issue of quantum teleportation, and information transfer.

All this is unknown, and requires extreme risk taking. Both experimentally 
and theoretically speaking.

To which the author wishes best wishes for those brave enough to sail to the edge and to explore what we think we know, but may have no idea of.

Finally, in the last section, the author answers the following question. I.e. are we referring to an actual black hole we create in the laboratory, or an induced physical system mimicking a black hole.

This is due to the query of a referee, to which we state unequivocally that we are talking of the former, and we refer readers to this very last section to ascertain some issues as to how we think of semi classical gravity, the possibility of black body generation of gravitons, and other issues. Note that the last reference, has several equations from Weinberg [24] where we state without reservation that the temperature of the black body cavity $T$, as given is the same as the temperature $T$ given in our main body for an induced real black hole. Finally it is useful to note that we are talking of nucleation of particles in space time, which is the last part of our answers to the referee which require significant derivational expansion of the theoretical issues we have started to investigate.

This is due to the query of a referee, to which we state unequivocally that we are talking of the former, and we refer readers to this last section to ascertain some issues as to how we think of semi classical gravity, the possibility of black body generation of gravitons, and other issues. Note that the last reference, has several equations from Weinberg [24] where we state without reservation that the temperature of the black body cavity $T$, as given is the same as the temperature $T$ given in our main body for an induced real black hole. Finally it is useful to note that we are talking of nucleation of particles in space time, which is the last part of the answers to the referee which require significant derivational expansion of the theoretical issues we have started to investigate. As well as how to avoid the problem of micro blackholes exploding, i.e. this is what we refer to in as the black hole bomb problem which we tie into a discussion of a violation of the no hair theorem. So then having introduced this, the conclusion of our ideas as given is followed by answers given to a referee who asked questions we will include in for the record.

\section{Answering Questions Which Were Raised by a Referee as to This Problem of an Induced Rotating Kerr-Newmann Black Hole}

On September 24, 2018 the author received the following from a referee as to this paper, as initially reviewed, and his remarks reference Weinberg [24].

Quote

I have read Beckwith's paper. The biggest problem or question I have is the extent to which he is talking about producing a real black hole. If not then he appears to be equating temperature with the $T=1 /(4 \pi(2 m-\omega))$ of a black hole emitting radiation at that temperature with the plasma physics under compression at that temperature. From there this is thought to generate gravitational 
radiation. Weinberg has a chapter largely devoted to fluctuations, and compression and generating gravitational radiation. I have a hard time seen the connection. Equations (10.8.9) through (10.8.13) seem to be of some relevance. I think some clarifying statements are in order to make it clear what this is. Some of this almost reads as if it is about generating a real black hole.

End of quote

Yes, it is to generate a REAL black hole.

Agree that, in principle, that this problem, also is similar to a black body radiation spectrum as given in page 287 which involves using the formula, and this is for $n(\omega)$ be the number of emitted gravitons from a region of space time, with surface area. A(Kerr-Newmann), as created, to give at first glance commonality with use of the formula given in [24], page 287, of

$$
n(\omega) \mathrm{d} \omega=\frac{\omega^{2} \mathrm{~d} \omega}{\pi^{2} \cdot\left[\exp \left(\frac{h \omega}{k_{B} \cdot T_{\text {temp }}}\right)-1\right]}
$$

One of the questions to answer, in subsequent investigations, is to what degree our induced by laser physics construction, in terms of absorbed power, is commensurate with a leakage of gravitons from an induced rotating Kerr black hole as given by Equation (10.8.9) to Equation (10.8.13) of page 287 of [24] with the provision that this is for a temperature $T_{\text {temp }}$ induced in a small region of space time most likely by focusing upon a small region of an induced plasma, Kilowatt lasers. I.e. this in itself would entail examining, the details of the formula are given by

$$
A(\omega) n(\omega) \mathrm{d} \omega=E(\omega) \mathrm{d} \omega
$$

With $A(\omega)$ being the absorption of energy, of the Kerr Newmann rotating black hole, and $A(\omega)$ most likely due to a graviton gas, with energies of the order of what one would expect from a black body cavity.

This is due to the idea of [110] being generalized, as introduced to the author by Glinka in 2007 in the conference in Kiev, about symmetries in mathematical physics, with the idea that indeed, Equation (37) and Equation (38) of a cavity, are to a good first order approximation, a working model which will be refined.

We submit upon doing this, that this idea involves, using $T_{\text {temp }}$ which is in common with the same temperature as given in the earlier part of this manuscript according to the ideas submitted by Corda and which was also discussed by Beckwith. I.e. that $T_{\text {temp }}$ is for the temperature of an induced Kerr Newmann black hole, and also of induced temperature from kilowatt lasers as applied to the formed black hole.

A lot of details need to be worked out by a detailed laser physics prospective study, but in passing we wish to make a certain number of statements.

In DICE 2018, the author witnessed two presentations by both Pisen Chen, and Alexander Burinskii. Burinskii asserted to the author in Dice 2018 that the term, J, for angular momentum would involve semi classical approximations, 
implying that in his view that gravity itself is semi classical, for reasons which showed up in [111] and [112], which was referenced in a presentation which showed up in DICE 2018.

Pisen Chen, had a model as to a pseudo black hole, in DICE 2018, which is in common with some issues which he brought up in [113], but a large part of the presentation was done in DICE 2018 with the idea of laser physics mimicking a black hole, not necessarily Kerr, which would mimic some of the features he and others wish to induce for study as to purported quantum effects.

Whereas Burinskii, in conversation with the author in DICE focused upon the semi classical nature of J, i.e. induced momentum, for the rotating black hole as giving a semi classical nature to gravity, Chen, in DICE 2018 talked of part of his presentation as involving a Bogoliubov transformation involving a delineation as to if or not gravitational effects from an induced artificial black hole could have a semi classical characteristic and for this the author refers to the references given in [114] [115] [116] [117] [118] as to a dividing line between semi classical to quantum effects which could be delineated.

In the case of the Chen presentation in DICE 2018, the key details of if there was a semi classical effect came from [119], i.e.

\section{Quote, from [119]}

The question of whether Hawking evaporation violates unitarity, and therefore results in the loss of information, has remained unresolved since Hawking's seminal discovery. To date, the investigations have remained mostly theoretical since it is almost impossible to settle this paradox through direct astrophysical black hole observations. Here, we point out that relativistic plasma mirrors can be accelerated drastically and stopped abruptly by impinging intense $x$-ray pulses on solid plasma targets with a density gradient. This is analogous to the late time evolution of black hole Hawking evaporation. A conception of such an experiment is proposed and a self-consistent set of physical parameters is presented. Critical issues, such as how the black hole unitarity may be preserved, can be addressed through the entanglement between the analog Hawking radiation photons and their partner modes.

\section{End of quote}

The process of the acceleration of the mirror, as indicated above, could indicate either a classical, semi classical or approaching to quantum conditions for gravitational radiation from an INDUCED pseudo black hole.

The primary difference between our approach and [119] is that Chen is referring to an INDUCED pseudo black hole, whereas we are referencing an actual MINI Kerr Newmann black hole.

Having said that, we are attempting through different means to accomplish the same goal.

Keep in mind that in [119] and also in DICE, that the so called "Phase velocity of the Plasma mirror" as induced by the set up given in [119] which can be written as 


$$
\ddot{x}_{n}=\ddot{x}_{g}-\ddot{\lambda}_{g}=v_{g}
$$

has a left hand side, which as Chen related to the Author in DICE 2018, is a Bogoliubov transformation, and this is for the velocity of a so called "Plasma mirror" as discussed in [119] which is a semi classical phenomena.

From there, Chen asserted that in [119] he and others are trying to build up a case for a semi classical rendition of gravity.

The synopsis is from [119] that: Plasma Mirror Mimics Evaporating Black Hole.

The results of this appears partly to have some commonality with [120] which has the following quote

\section{Quote, from the abstract}

We examine the creation of particles, and more generally the transformation of quantum field states, due to boundary motion in curved spacetime. We provide a novel method enabling the calculation of the effect for a wide range of trajectories and spacetimes. We apply this to the experimental scenario used to detect the DCE, now adopting the Schwarzschild metric, and find novel resonances in particle creation as a result of the spacetime curvature.

In a word, this idea is a way, via the Bogoliubov transformation to initiate creation of particles from space time, and we assert that in doing so, that the particle creation, may be due to either semi classical regimes of "particle creation" which maybe akin to the result given in page four of [120] which has the following quote, which has what they called [46] as our [100] and what they called [25] in [120] as:

We now consider the cavity to be embedded in some curved spacetime, and assume that the latter admits a timelike Killing vector field in the region of interest, so that we can construct a well-defined Hilbert space from the solutions to the field equation [46]. It is always possible to find some coordinate system in which the metric is conformally flat [25], and consequently the Klein-Gordon equation takes the same form as in inertial coordinates in flat space. Letting $(t, x)$ now denote these conformally flat coordinates, one finds that the framework above, in particular Equations (1)-(3), holds.

In essence the semi classical nature of the process is revealed in the allusion as to the creation of particles due to a "Klein Gordon equation" which is embedded in a Schwartzhield metric. As to the above, what is called reference [46] we will list as [100] as given by Wald and quantum curved space time.

Much the same thing could be realized up in part as to investigating the physics of [118] and [120] of our reference list which would in turn be akin to using [120] of our references as a way to re phrase what we are imagining this problem to be in terms of information theory.

In all, the main end result is to avoid the so called black hole bomb effect.

Much the same thing could be realized up in part as to investigating the physics of [119] and [120] of our reference list which would in turn be akin to using [121] of our references as a way to re phrase what we are imagining this problem 
to be in terms of information theory. It also overlaps with Wald's work as of 1984 as given by [54].

In all, the main end result is to try to avoid the so called black hole bomb effect, where a mini black hole would explode in a laboratory setting within say $10^{\wedge}-16$ or so seconds, I.e. the idea would be to have a reasonably stable configuration within put laser energy, but a small mass, and to do it over hopefully $10^{\wedge} 15$ or more times longer than the $10^{\wedge}-16$ second where the mini black hole would quickly evaporate. I.e. a duration of say up to $10^{\wedge}-1$ seconds which would provide a base line as to astrophysical modeling of a Kerr-Newman black hole.

The idea is, in fact that we have three regimes of input into our system.

The first input is from the lasers, or possibly an underground nuclear explosion. I.e. most likely involving Gigawatt lasers.

Secondly if the input energy were pulsed in, of an ellipsoidal regime of space time commensurate with the Kerr-Newman rotating black hole.

Third, due to the black hole bomb effect, of dumping of output from the rotating Kerr-Newman black hole.

It is important to stress that in all of this we are assuming a defacto mini black hole, not a simulated black hole.

This would be at the minimum an awesomely complicated, very well done balancing at, which would permit the generation of gravitational radiation, I.e. hopefully as detectible gravitons.

I.e. the balancing act would entail stretching out the life of the mini black hole from about $10^{\wedge}-16$ seconds to about $10^{\wedge}-1$ seconds.

To say this would involve superb laser engineering is an understatement, I.e. if this were done in the Laser lab at Lawrence Livermore labs in the ignition facility, it would be the most delicate piece of ignition physics ever achieved.

We argue that the rewards for this superb balancing act may allow a quantification of experimental tests which could clarify if Gravity is either classical or quantum in its foundations. I.e. this is very much worth doing.

Much the same thing could be realized up in part as to investigating the physics of [119] and [120] of our reference list which would in turn be akin to using [120] of our references as a way to re phrase what we are imagining this problem to be in terms of information theory. It also overlaps with Wald $s$ work as of 1984 as given by [54]. In all, the main end result is to try to avoid the so called black hole bomb effect, where a mini black hole would explode in a laboratory setting within say $10^{\wedge}-16$ or so seconds, I.e. the idea would be to have a reasonably stable configuration within put laser energy, but a small mass, and to do it over hopefully $10^{\wedge} 15$ or more times longer than the $10^{\wedge}-16$ second where the mini black hole would quickly evaporate. I.e. a duration of say up to $10^{\wedge}-1$ seconds which would provide a base line as to astrophysical modeling of a Kerr-Newman black hole. Keep in mind that this idea of avoiding a black hole bomb has a long history, i.e. The first discussion of a runaway effect, the black hole bomb, was explored by. Press and Teukolsky in 1972 [122]. 
First of all, would be to determine, if the mini black hole bomb, would spontaneously occur, unless the Kerr-Newmann black hole were carefully engineered in the laboratory.

Secondly if such an effect was to occur, it may signify new physics beyond the Standard Model, i.e. a spontaneous blow up which we would need to counter.

It also would link to the idea that black holes have "hair", as pointed out by a paper from 2017, by East and Pretorius [123].

In all, the idea, once again, is how to create conditions which conceivably could lead to a spontaneous black hole bomb, if not carefully calibrated, and how to fix this problem via careful use of laser physics.

Doing so would allow us to delve deeply into both gravitational physics, quantum mechanics, and also the nature of if or not quantum gravity could be ascertained in a laboratory setting.

\section{Acknowledgements}

This work is supported in part by National Nature Science Foundation of China grant No. 11375279.

\section{Conflicts of Interest}

The authors declare no conflicts of interest regarding the publication of this paper.

\section{References}

[1] Ohanian, H. and Ruffini, R. (2013) Gravitation and Spacetime. 3rd Edition, Cambridge University Press, New York City. https://doi.org/10.1017/CBO9781139003391

[2] Kuchiev, M. (1994) Polarization of Instantons and Gravity. Europhysics Letters, 28, 539-544. https://arxiv.org/abs/hep-th/9411090 https://doi.org/10.1209/0295-5075/28/8/001

[3] Müller, R. and. Lousto, C. (1995) Entanglement Entropy in Curved Spacetimes with Event Horizons. Physical Review D, 52, 4512.

https://arxiv.org/pdf/gr-qc/9504049.pdf https://doi.org/10.1103/PhysRevD.52.4512

[4] Jack, N.Y. (2007) Holographic Foam, Dark Energy and Infinite Statistics. Physics Letters B, 657, 10-14. https://doi.org/10.1016/j.physletb.2007.09.052

[5] Jack, N.Y. (2008) Article: Spacetime Foam: From Entropy and Holography to Infinite Statistics and Nonlocality. Entropy, 10, 441-461。 https://doi.org/10.3390/e10040441

[6] Ezra, N. and Allen, J. (1965) Note on the Kerr Spinning-Particle Metric. Journal of Mathematical Physics, 6, 915-917. https://doi.org/10.1063/1.1704350

[7] Ezra, N., Couch, E., Chinnapared, K., Exton, A., Prakash, A. and Torrence, R. (1965) Metric of a Rotating, Charged Mass. Journal of Mathematical Physics, 6, 918-919. https://doi.org/10.1063/1.1704351

[8] Kerr, R.P. (1963) Gravitational Field of a Spinning Mass as an Example of Algebraically Special Metrics. Physical Review Letters, 11, 237-238. 
https://doi.org/10.1103/PhysRevLett.11.237

[9] Kerr, R. (2009) The Kerr and Kerr-Shield Metrics. In: Wilkshire, D., Visser, M. and Scott, S., The Kerr Spacetime, Rotating Black Holes in General Relativity, Cambridge University Press, New York City, 38-72.

[10] Citation Number Five on Page 400 Called (Appell, Math. Ann. xxx (1887)) (1996) Discussed by Wittaker, E. and Watson, G., A Course of Modern Analysis, Cambridge University Press, Cambridge, 155-156.

[11] Hackmann, E. and $\mathrm{Xu}, \mathrm{H}$. (2003) Charged Particle Motion in Kerr-Newmann Space-Times. https://arxiv.org/pdf/1304.2142.pdf\#page $=4$

[12] Plebasnki, J. and Krasinki, A. (2007) An Introduction to General Relativity and Cosmology. Cambridge University Press, Cambridge.

[13] Thorne, K. and Blandford, R. (2017) Modern Classical Physics. Princeton University Press, Princeton.

[14] Ryden, B. (2017) Introduction to Cosmology. 2nd Edition, Cambridge University Press, Cambridge.

[15] Reichl, L. (1980) Modern Course in Statistical Physics. University of Texas Press, Austin.

[16] Narlikar, J. (1983) Introduction to Cosmology. Jones and Barlett Publishers, Boston, MA.

[17] Penrose, R. (1989) Difficulties with Inflationary Cosmology. Annals of the New York Academy of Sciences, 571, 249-264. https://doi.org/10.1111/j.1749-6632.1989.tb50513.x

[18] Kieffer, C.L. (2001) Entropy of Gravitational Waves and Primordial Fluctuations. AIP Conference Proceedings, 555, 499-504,

[19] Park, D. (1955) Radiations from a Spinning Rod. Physical Review, 99, 1324-1325. https://doi.org/10.1103/PhysRev.99.1324

[20] Schneider, C. and Lippert, T. (2010) Laser Ablation and Thin Film Deposition. In: Schaaf, P., Ed., Laser Processing of Materials, Springer Series in Materials Science, Vol. 139, Springer, Berlin.

[21] Schoenlein, R., et al. (2000) Generation of Femtosecond X-Ray Pulses via Laser-Electron Beam Interaction. Applied Physics B, 71, 1-10.

https://doi.org/10.1007/PL00021152

[22] Beckwith, A. (2017) Variation in Gravity Due to Laser Inducing an Explosion-Comparison with the Spinning Barbell Driven by Lasers. Journal of High Energy Physics, Gravitation and Cosmology, 3, 558-563.

https://doi.org/10.4236/jhepgc.2017.34042

[23] Lightman, A., Press, W., Price, R. and Teukolsky, S. (1975) Problem Book in Relativity and Gravitation. Princeton University Press, Princeton, NJ.

[24] Weinberg, S. (1972) Gravitation and Cosmology, Principles and Applications of the General Theory of Relativity. John Wiley \& Sons, Inc., New York.

[25] Baker Jr., R.M.L. (2006) Novel Formulation of the Quadrupole Equation for Potential Stellar Gravitational-Wave Power Estimation. Astronomische Nachrichten/Astronomical Notes, 327, 710-713.

http://www.drrobertbaker.com/docs/Astron.\%20Notes\%20GW\%20Article.pdf https://doi.org/10.1002/asna.200510617

[26] Beckwith, A. and Baker, R. (2015) The Generation of Gravitational Waves by Lasers. Proceedings of the Space Technology \& Applications International Forum 
(STAIF II 2015). Albuquerque, New Mexico, 16-18 April 2015, 1-12.

[27] Rindler, W. (1977) Essential Relativity, Special, General, and Cosmological. 2nd Edition, Springer Verlag, New York, NY, USA.

http://202.38.64.11/ jmy/documents/ebooks/Rindler\%202006\%20Relativity\%20(IS BN\%2044198567324).pdf

[28] Peebles, P. (1993) Principles of Physical Cosmology. Princeton University Press, Princeton, NJ.

[29] Beckwith, A.W. (2017) Part 2: Review of Tokamak Physics to Construct a Device Optimal for Graviton Detection and Generation within a Confined Small Spatial Volume, as Opposed to Dyson's "Infinite Astrophysical Volume" Calculations. Journal of High Energy Physics, Gravitation and Cosmology, 3, 138-155. https://doi.org/10.4236/jhepgc.2017.31015

[30] Beckwith, A.W. (2016) Open Question: Could a Causal Discontinuity Explain Fluctuations in the CMBR Radiation Spectrum? Journal of High Energy Physics, Gravitation and Cosmology, 2, 186-208. https://doi.org/10.4236/jhepgc.2016.22018

[31] Valtonen M. and Karttunen, H. (2005) The Three-Body Problem. Cambridge University Press, New York, NY.

[32] Beckwith, A.W. (2018) Structure Formation in the Early Universe as a Result of Non Linear Electrodynamics Influencing Scale Factor Size with Attendant Changes in Gravitational Potential and Its Relationship to the 3 Body Problem. http://vixra.org/pdf/1805.0357v1.pdf

[33] Abraham, R. and Marsden, J. (1985) Mechanics. 2nd Edition, Addison and Wesley, Menlo Park, CA.

[34] Calmet, X., Carr, B. and Winstanley, W. (2014) Quantum Black Holes. Springer Verlag, New York, NY. https://doi.org/10.1007/978-3-642-38939-9

[35] Camara, C.S., de Garcia Maia, M.R., Carvalho, J.C. and Lima, J.A.S. (2004) Nonsingular FRW Cosmology and Non Linear Dynamics.

http://arxiv.org/pdf/astro-ph/0402311.pdf

[36] Corda, C. and Cuesta, H. (2010) Removing Black Hole Singularities with Non Linear Electrodynamics. Modern Physics A, 25, 2423-2429

[37] Freeze, K. (1992) Inflation. In: Nath, P. and Reucroft, S., Eds., Particles, Strings, and Cosmology, World Scientific, Singapore, 408-424.

[38] Maggiore, M. (2018) Gravitational Waves, Volume 2: Astrophysics and Cosmology. Oxford University Press, Oxford, UK.

[39] Whittaker, E. (1989) A History of the Theories of Aether and Electricity, Two Volumes Bound as One, Volume 1: Classical Theories, and Volume 2: The Modern Theories, 1900 to 1926. Dover Publications, Inc., Mineola, NY.

[40] Alder, R., Bazin, M. and Shiffer, M. (1975) Introduction to General Relativity. 2nd Edition, McGraw-Hill Book Company, San Francisco, CA.

[41] Hajicek, P., et al. (2008) An Introduction to the Relativistic Theory of Gravitation. Springer, Beilin, 243.

[42] Astorino, M., Compere, Oliveri, R., Vandevoorde, N. and Vandevoorde, N. (2016) Mass of Kerr-Newman Black Holes in an External Magnetic Field. https://arxiv.org/pdf/1602.08110.pdf

[43] Christodoulou, D. and Ruffini, R. (1971) Reversible Transformations of a Charged Black Hole. Physical Review D, 4, 3552-3555.

https://doi.org/10.1103/PhysRevD.4.3552 
[44] Thorne, K., Price, R., MacDonald, D., Suen, W. and Zhang, X. (1986) Rapidly Rotating Black Holes. In: Thorne, K., Price, R. and MaDonald, D., Eds., Black Holes, the Membrane Paradigm, Yale University Press, New Haven, Connecticut, 67-120.

[45] Yarmana, T., Kholmetskii, A., Yarman, O., Arikd, M. and Yarman, F. (2018) Second Law of Thermodynamics Is Ingrained within Quantum Mechanics. Results in Physics, 10, 818-821. https://doi.org/10.1016/j.rinp.2018.06.058

[46] Hartnett, K. (2018) Mathematicians Disprove Conjecture Made to Save Black Holes. https://www.quantamagazine.org/mathematicians-disprove-conjecture-made-to-sa ve-black-holes-20180517/

[47] Dafermos, M. and Luk, J. (2017) The Interior of Dynamical Vacuum Black Holes I: The $C^{0}$-Stability of the Kerr Cauchy Horizon. https://arxiv.org/abs/1710.01722

[48] Lloyd, S. (2002) Computational Capacity of the Universe. Physical Review Letters, 88, Article ID: 237901. https://doi.org/10.1103/PhysRevLett.88.237901

[49] Roger, P. (1994) The Question of Cosmic Censorship. In: Wald, R., Ed., Black Holes and Relativistic Stars, University of Chicago Press, Chicago, IL.

[50] Roger, P. (1979) Singularities and Time-Asymmetry. In: Hawking and Israel, Eds., General Relativity: An Einstein Centenary Survey, Cambridge University Press, Cambridge, 617-629.

[51] Shapiro, S.L. and Teukolsky, S.A. (1991) Formation of Naked Singularities: The Violation of Cosmic Censorship. Physical Review Letters, 66, 994-997.

https://doi.org/10.1103/PhysRevLett.66.994

[52] Robert, W. (1984) General Relativity. 299-308.

[53] John, N. (1998) Early Steps toward Inertial Fusion Energy (IFE). LLNL.

[54] Stolz, C. (2007) The National Ignition Facility. The World'S Largest Optical System. In: Wang, Y.T., Tschudi, T.T., Rolland, J.P. and Tatsuno, K., Eds., Optical Design and Testing III, Vol. 6834, Optics and Photonics News, Washington DC.

[55] Lynda, S. and Bob, H. (2009) NIF's Future Ignites with 192-Beam Shot. Lawrence Livermore National Laboratory.

[56] van der Vink, G.E. (1989) The Containment of Underground Nuclear Explosions. U.S. Congress, Office of Technology Assessment, OTA-ISC-414.

[57] Teller, E., Talley W., Gary, H., Higgins, G. and Johnson, G. (1968) The Constructive Uses of Nuclear Explosives. McGraw-Hill, New York, NY.

[58] Ray, A. (2004) Stars as Thermonuclear Reactors: Their Fuels and Ashes. https://arxiv.org/abs/astro-ph/0405568

[59] Reiners, A. and Basri, G. (2009) On the Magnetic Topology of Partially and Fully Convective Stars. Astronomy and Astrophysics. 496, 787-790.

https://arxiv.org/abs/0901.1659 https://doi.org/10.1051/0004-6361:200811450

[60] Crowell, L. (2017) Personal Note.

[61] Corda, C. (2011) Effective Temperature for Black Holes. Journal of High Energy Physics, 2011, Article No. 101. https://doi.org/10.1007/JHEP08(2011)101

[62] Corda, C., Hendi, S.H., Katebi, R. and Schmidt, N.O. (2014) Hawking Radiation-Quasi-Normal Modes Correspondence and Effective States for Nonextremal Reissner-Nordstrom Black Holes. Advances in High Energy Physics, 2014, Article ID: 527874. https://doi.org/10.1155/2014/527874

[63] Corda, C. (2012) Effective Temperature, Hawking Radiation and Quasinormal Modes. International Journal of Modern Physics D, 21, Article ID: 1242023. 
https://arxiv.org/abs/1205.5251 https://doi.org/10.1142/S0218271812420230

[64] Corda, C., Hendi, S.H., Katebi, R. and Schmidt, N.O. (2013) Effective State, Hawking Radiation and Quasi-Normal Modes for Kerr Black Holes. Journal of High Energy Physics, 2013, Article No. 008. https://doi.org/10.1007/JHEP06(2013)008

[65] Corda, C. (2013) Non-Strictly Black Body Spectrum from the Tunneling Mechanism. Annals of Physics, 337, 49-54. https://doi.org/10.1016/j.aop.2013.05.006

[66] Sun, D.-Q., Wang, Z.-L., He, M., Hu, X.-R. and Deng, J.-B. (2017) Hawking Radiation-Quasinormal Modes Correspondence for Large AdS Black Holes. Advances in High Energy Physics, Article ID: 4817948. https://arxiv.org/ftp/arxiv/papers/1708/1708.06869.pdf

[67] Tipler, F.J., Clarke, C.J.S. and Ellis, G.F.R. (1980) Singularities and Horizons: A Review Article, In: Held, A., Ed., General Relativity and Gravitation: One Hundred Years after the Birth of Albert Einstein, Plenum Press, New York, 97.

[68] Stephen, H. and Ellis, G.F.R. (1973) The Large Scale Structure of Space-Time. Cambridge University Press, Cambridge.

[69] Gödel, K. (1949) An Example of a New Type of Cosmological Solutions of Einstein's Field Equations of Gravitation. Reviews of Modern Physics, 21, 447. https://doi.org/10.1103/RevModPhys.21.447

[70] Roger, P. (1969) Gravitational Collapse: The Role of General Relativity. La Rivista del Nuovo Cimento, 1, 252-276.

[71] Hans, S., Dietrich, K., Malcolm, M., Cornelius, H. and Eduard, H. (2003) Exact Solutions to Einstein's Field Equations. 2nd Edition, Cambridge University Press, Cambridge, UK.

[72] Crowell, L. (2018) Personal Note.

[73] Haliday, D., Resnick, R. and Walker, J. (2001) Fundamentals of Physics. Vol. 1, 6th Edition, John Wiley and Sons, Hoboken, NY.

[74] Beckwith, A.W. (2016) Linkage of Classical Mechanical (CM) Geometry (3 Dimensional) and Quantum Mechanical (QM) Geometry (2 Dimensional) via Hopf Mapping and Its Implications for Relic Gravitational Wave (GW) Power Production. Journal of High Energy Physics, Gravitation and Cosmology, 2, 447-456. https://doi.org/10.4236/jhepgc.2016.24038

[75] Borsten, G.L., Duff, M. and Levay, P. (2012) The Black Hole/Qubit Correspondence: An Up-to-Date Review. Classical and Quantum Gravity, 29, Article ID: 224008.

[76] Beckwith, A. (2015) How Graviton Power Values and a Graviton Count from the Electroweak Era, Give Strain and Heavy Gravity Values. In: Dumarchez, J., Fontaine, G., Klima, B. and Van, J.T.T., Eds., The Inaugural Confrence on ICISE "Windows to the Universe" under the Patronage of "Annee France-Vietnam 2013-2014" under the High Patronage of UNESCO, The Gioi Publishers, Hanoi, 411-412. http://vixra.org/abs/1308.0110

[77] Cardoso, V., Dias, O.J.C., Jose, P.S. and Lemos, S.Y. (2004) The Black Hole Bomb and Superradiant Instabilities. Physical Review D, 70, Article ID: 044039.

https://arxiv.org/abs/hep-th/0404096 https://doi.org/10.1103/PhysRevD.70.044039

[78] Li, F.Y., Baker Jr., R.M.L., Fang, Z.Y., Stephenson, G.V. and Chen, Z.Y. (2008) Perturbative Photon Fluxes Generated by High-Frequency Gravitational Waves and Their Physical Effects. The European Physical Journal C, 56, 407-423. https://arxiv.org/abs/0806.1989 
https://doi.org/10.1140/epjc/s10052-008-0656-9

[79] Dowker, F. (2005) Causal Sets and the Deep Structure of Space-Time. In: Akshtekar, A., Ed., 100 Years of Relativity, Space-Time Structure: Einstein and Beyond, with editor, World Press Scientific, P.T.E., Singapore, 445-464.

https://arxiv.org/abs/gr-qc/0508109

[80] Barvinsky, A., Kamenschick, A. and Yu, A. (2006) Thermodynamics from Nothing: Limiting the Cosmological Constant Landscape. Physical Review D, 74, Article ID: Article ID: 121502.

[81] Beckwith, A.W. (2016) Can Thermal Input from a Prior Universe Account for Relic Graviton Production? Implications for the Cosmological Landscape. Journal of High Energy Physics, Gravitation and Cosmology, 2, 344-361. https://doi.org/10.4236/jhepgc.2016.23032

[82] Beckwith, A.W. (2014) Analyzing Black Hole Super-Radiance Emission of Particles/Energy from a Black Hole as a Gedanken Experiment to Get Bounds on the Mass of a Graviton. Advances in High Energy Physics, 2014, Article ID: 230713. https://doi.org/10.1155/2014/230713

[83] Walcott, B.A. (2016) Geddanken Experiment for Quark Star Idea, Quantum Wavelength Limit, Minimum Time, and Early Universe Temperature, from First Principles. Journal of High Energy Physics, Gravitation and Cosmology, 2, 478-485.

[84] Walcott, B.A. (2016) Using a Multiverse Version of Penrose Cyclic Conformal Cosmology to Obtain Ergodic Mixing Averaging of Cosmological Information Transfer to Fix H Bar (Planck's Constant) in Each New Universe Created during Recycling of Universes Due to CCC, Multiverse Style. Journal of High Energy Physics, Gravitation and Cosmology, 2, 506-511.

[85] Walcott, B.A. (2016) Examination of a Multiple Universe Version of the Partition Function of the Universe, Based upon Penrose's Cyclic Conformal Cosmology. Leading to Uniform Values of h Bar (Planck's Constant) and Invariant Physical Laws in Each Universe of the "Multiverse". Journal of High Energy Physics, Gravitation and Cosmology, 2, 571-580.

[86] Walcott, B.A. (2017) Pre-Octonionic to Octonionic Gravity and Could Kinetic Energy Be Larger than Potential Energy before Inflation? Journal of High Energy Physics, Gravitation and Cosmology, 3, 693-707.

[87] Walcott, B.A. (2017) The Transition from Pre-Octonionic to Octonionic Gravity and How It May Be Pertinent to a Re-Do of the HUP for Metric Tensors. Journal of High Energy Physics, Gravitation and Cosmology, 3, 727-753.

[88] Abbott, B. P., et al. (2016) Observation of Gravitational Waves from a Binary Black Hole Merger. Physical Review Letters, 116, Article ID: 061102.

[89] Abbott, B.P., et al. (2016) GW151226: Observation of Gravitational Waves from a 22-Solar-Mass Binary Black Hole Coalescence. Physical Review Letters, 116, Article ID: 241103 .

[90] Abbott, B. P., et al. (2017) GW170104: Observation of a 50-Solar-Mass Binary Black Hole Coalescence at Redshift 0.2. Physical Review Letters, 118, Article ID: 221101. https://doi.org/10.1103/PhysRevLett.118.221101

[91] Corda, C. (2009) Interferometric Detection of Gravitational Waves: The Definitive Test for General Relativity. International Journal of Modern Physics D, 8, 2275-2282. https://arxiv.org/abs/0905.2502 https://doi.org/10.1142/S0218271809015904

[92] Barcelo, C. and Visser, M. (1999) Transversable Wormholes from Massless Con- 
formally Coupled Scalar Fields. Physics Letters B, 466, Article ID: 15123.

[93] Visser, M. (2002) Sakarov’s Induced Gravity: A Modern Perspective. https://arxiv.org/abs/gr-qc/0204062

[94] Sterman, G. and Yitp, S.B. () Introduction to the Parton Model and Perturbative QCD.

http://www.hep.wisc.edu/cteq11/lectures/sterman_CQ11S_part_I_07.10.11.pdf

[95] Mueller, A.H. (1989) Perturbative Quantum Chromodynamics. World Scientific, Singapore.

[96] Greene, B., Samir, D., Mathur, S. and O'Neill, C. (1993) Eluding the No-Hair Conjecture: Black Holes in Spontaneously Broken Gauge Theories. Physical Review D, 47, 2242-2259. https://arxiv.org/abs/hep-th/9211007 https://doi.org/10.1103/PhysRevD.47.2242

[97] Crowell, L. (2018) In Private E-Mail to the Author.

[98] Coleman, S., Preskill, J. and Wilczek, F. (1992) Quantum Hair on Black Holes. Nuclear Physics B, 378, 175-246. https://arxiv.org/abs/hep-th/9201059 https://doi.org/10.1016/0550-3213(92)90008-Y

[99] Bhattacharya, S. and Lahiri, A. (2007) No Hair Theorems for Positive $\Lambda$. Physical Review Letters, 99, Article ID:201101. https://arxiv.org/abs/gr-qc/0702006

[100] Wald, R.M. (1984) General Relativity. The University of Chicago Press, Chicago, 312-324. https://doi.org/10.7208/chicago/9780226870373.001.0001

[101] Park, D.K., Kim, H. and Tamarayan, S. (2002) Nonvanishing Cosmological Constant of Flat Universe in Brane World Senarios. Physics Letters B, 535, 5-10. https://doi.org/10.1016/S0370-2693(02)01729-X

[102] Misner, C.W., Thorne, K.S., Estate of Wheeler, Archibald, J. (2017) Gravitation. Princeton University Press, Princeton, NJ, 863.

[103] Carroll, S. and Chatwin-Davies, A. (2017) Cosmic Equilibration: A Holographic No-Hair Theorem from the Generalized Second Law. Physical Review D, 97, Article ID: 046012. https://arxiv.org/abs/1703.09241

[104] Mavromatos, N. (1996) Eluding the No-Hair Conjecture for Black Holes. Oxford. https://arxiv.org/abs/gr-qc/9606008

[105] Heusler M. (1996) No-Hair Theorems and Black Holes with Hair. https://cds.cern.ch/record/312731/files/9610019.pdf

[106] Israel, W. (1967) Event Horizons in Static Vacuum Space-Times. Physical Review Journals Archive, 164, 1776. https://doi.org/10.1103/PhysRev.164.1776

[107] Hajicek, P. (2008) An Introduction to the Relativistic Theory of Gravitation. Lecture Notes in Physics, Vol. 750, Springer Verlag, Berlin.

https://doi.org/10.1007/978-3-540-78659-7

[108] Beckwith, A.W. (2018) Refining Black Hole Physics to Obtain Planck's Constant from Information Shared from Cosmological Cycle to Cycle (Avoiding Super-Radiance).

https://www.researchgate.net/publication/327562281_Refining_black_hole_physics _to_obtain_Planck's_constant_from_information_shared_from_cosmological_cycle _to_cycle_avoiding_super-radianc

[109] Beckwith, A.W. (2016) Using a Multiverse Version of Penrose Cyclic Conformal Cosmology to Obtain Ergodic Mixing Averaging of Cosmological Information Transfer to Fix H Bar (Planck's Constant) in Each New Universe Created during Recycling of Universes Due to CCC, Multiverse Style. Journal of High Energy Physics, Gravitation and Cosmology, 2, 506-511.

https://doi.org/10.4236/jhepgc.2016.24044 
[110] Glinka, L. (2007) Quantum Information from Graviton-Matter Gas. SIGMA, 3, Article No. 087. https://arxiv.org/abs/0707.3341

[111] Burinskii, A. (2014) Gravitating Bag as a Coherent System of the Point-Like and Dressed Electron. https://arxiv.org/abs/1502.00736

[112] Burinskii, A. (2015) Emergence of the Dirac Equation in the Solitonic Source of the Kerr Spinning Particle. Gravitation and Cosmology, 21, 28. https://arxiv.org/abs/1404.5947 https://doi.org/10.1134/S020228931501003X

[113] Chen, P., Ong, Y.C. and Yeom, D. (2015) Black Hole Remnants and the Information Loss Paradox. Physics Reports, 1-45. https://arxiv.org/abs/1412.8366 https://doi.org/10.1016/j.physrep.2015.10.007

[114] Boboliubov, N.N. (1958) A New Method in the Theory of Superconductivity. I. Soviet Physics (U.S.S.R.). JETP, 7, 41-46.

[115] Bogoliubov, N.N. (1958) A New Method in the Theory of Superconductivity III. (PDF). Soviet Physics (U.S.S.R.). JETP, 34, 51-55.

[116] Bogolyubov, N.N., Tolmachev, V.V. and Shirkov, D.V. (1958) A New Method in the Theory of Superconductivity. Fortschitte der Physik, 6, 605-682. https://doi.org/10.1002/prop.19580061102

[117] Strutinsky, V.M. (1967) Shell Effects in Nuclear Masses and Deformation Energies. Nuclear Physics A., 95, 420-442. https://doi.org/10.1016/0375-9474(67)90510-6

[118] Svozil, K. (1990) Squeezed Fermion States. Physical Review Letters, 65, 3341-3343. https://doi.org/10.1103/PhysRevLett.65.3341

[119] Chen, P. and Mourou, G. (2017) Accelerating Plasma Mirrors to Investigate the Black Hole Information Loss Paradox. Physical Review Letters, 118, Article ID: 045001 https://doi.org/10.1103/PhysRevLett.118.045001

[120] Lock, M. and Fuentes, I. (2017) Dynamical Casimir Effect in Curved Spacetime. New Journal of Physics, 19, Article ID: 073005. http://iopscience.iop.org/article/10.1088/1367-2630/aa7651

[121] Benenti, G., D’Arrigo, A., Siccardi, S. and Strini, G. (2014) Dynamical Casimir Effect in Quantum-Information Processing. Physical Review A, 90, Article ID: 052313. https://doi.org/10.1103/PhysRevA.90.052313

[122] Press, W.H. and Teukolsky, S.A. (1972) Floating Orbits, Superradiant Scattering and the Black-Hole Bomb. Nature, 238, 211-212. https://doi.org/10.1038/238211a0

[123] East, W. and Pretorius, F. (2017) Superradiant Instability and Backreaction of Massive Vector Fields around Kerr Black Holes. Physical Review Letters, American Physical Society. https://arxiv.org/abs/1704.04791 